\title{
The Cocoon nebula and its ionizing star: do stellar and nebular abundances agree? ${ }^{\star}$
}

\author{
J. García-Rojas ${ }^{1,2}$, S. Simón-Díaz ${ }^{1,2}$, and C. Esteban ${ }^{1,2}$ \\ ${ }^{1}$ Instituto de Astrofísica de Canarias, 38200 La Laguna, Tenerife, Spain \\ e-mail: jogarcia@iac.es \\ 2 Universidad de La Laguna, Dept. Astrofísica, 38204 La Laguna, Tenerife, Spain
}

Received 23 July 2014 / Accepted 29 September 2014

\section{ABSTRACT}

\begin{abstract}
Context. Main-sequence massive stars embedded in an H II region should have the same chemical abundances as the surrounding nebular gas+dust. The Cocoon nebula (IC 5146), a close-by Galactic H II region ionized by a narrow line B0.5 V single star $(\mathrm{BD}+463474)$, is an ideal target to compare nebular and stellar abundances in detail in the same Galactic region.

Aims. We investigate the chemical content of oxygen and other elements in the Cocoon nebula from two different points of view: an empirical analysis of the nebular spectrum, and a detailed spectroscopic analysis of the associated early B-type star using state-ofthe-art stellar atmosphere modeling. By comparing the stellar and nebular abundances, we aim to indirectly address the long-standing problem of the discrepancy found between abundances obtained from collisionally excited lines and optical recombination lines in photoionized nebulae.

Methods. We collected long-slit spatially resolved spectroscopy of the Cocoon nebula and a high-resolution optical spectrum of the ionizing star. Standard nebular techniques along with updated atomic data were used to compute the physical conditions and gaseous abundances of $\mathrm{O}, \mathrm{N}$, and $\mathrm{S}$ in eight apertures extracted across a semidiameter of the nebula. We performed a self-consistent spectroscopic abundance analysis of BD+46 3474 based on the atmosphere code FASTWIND to determine the stellar parameters and Si, O, and $\mathrm{N}$ abundances.

Results. The Cocoon nebula and its ionizing star, located at a distance of $800 \pm 80 \mathrm{pc}$, have a chemical composition very similar to the Orion nebula and other B-type stars in the solar vicinity. This result agrees with the high degree of homogeneity of the presentday composition of the solar neighborhood (up to $1.5 \mathrm{Kpc}$ from the Sun) as derived from the study of the local cold-gas interstellar medium. The comparison of stellar and nebular collisionally excited line abundances in the Cocoon nebula indicates that $\mathrm{O}$ and $\mathrm{N}$ gas+dust nebular values agree better with stellar values assuming small temperature fluctuations on the order of those found in the Orion nebula $\left(t^{2}=0.022\right)$. For $\mathrm{S}$, the behaviour is somewhat puzzling, and different conclusions can be reached depending on the atomic data set used.
\end{abstract}

Key words. H II regions - ISM: individual objects: IC 5146 - ISM: abundances - stars: abundances - stars: early-type stars: individual: BD+46 3474

\section{Introduction}

In nebular astrophysics, the oxygen abundance is the most widely used proxy of metallicity from the Milky Way to fardistant galaxies. A precise knowledge of its abundance as well as of nitrogen, carbon, $\alpha$-elements, and iron-peak elements and their ratios at different redshifts are crucial for understanding the nucleosynthesis processes in the stars and the chemical evolution history of the Universe (Henry et al. 2000; Chiappini et al. 2003; Carigi et al. 2005). Uncertainties in the knowledge of the oxygen abundance (i.e., metallicity) have important implications in several topics of modern astrophysics such as the luminosity- and mass-metallicity relations for local and highredshift star-forming galaxies (Tremonti et al. 2004), the calibrations of strong line methods for deriving the abundance scale of extragalactic $\mathrm{H}$ II regions and star-forming galaxies at different redshifts (Peimbert et al. 2007; Peña-Guerrero et al. 2012;

* Based on observations made with the William Herschel Telescope operated by the Isaac Newton Group and with the Nordic Optical Telescope, operated by the Nordic Optical Telescope Scientific Association. Both telescopes are at the Observatorio del Roque de los Muchachos, La Palma, Spain, of the Instituto de Astrofísica de Canarias.
López-Sánchez et al. 2012), or the determination of the primordial helium abundance (Peimbert 2008).

Ionized nebulae have always been claimed to be the most reliable and straightforward astrophysical objects to determine abundances from close-by to large distances in the Universe. However, they are not free from some difficulties. One of the most longstanding problems is the dichotomy systematically found between the nebular abundances provided by the standard method, based on the analysis of intensity ratios of collisionally excited lines (CELs, which strongly depend on the assumed physical conditions in the nebula) and the abundances given by the faint optical recombination lines (ORLs, which are almost insensitive to the adopted physical conditions).

As already pointed out in the pioneering work by Wyse (1942) in the planetary nebula NGC 7009 more than seventy years ago, the observational evidence of CEL and ORL providing discrepant results has increased significantly in the past decade, both using Galactic H II region data (Esteban et al. 2005, 2013; García-Rojas \& Esteban 2007, and references therein) and extragalactic H II region data (Esteban et al. 2002, 2009, 2014; Peimbert 2003; López-Sánchez et al. 2007; Peña-Guerrero et al. 2012). In particular, these authors have found that the $\mathrm{O}^{2+} / \mathrm{H}^{+}$ratio computed from O II ORLs gives sistematically 
higher values than the ratio obtained from [O III] CELs by a factor ranging $\sim 1.2-2.2$. Similar discrepancies have been reported for other ions for which abundances can be determined using both CELs and ORLs: $\mathrm{C}^{2+}, \mathrm{Ne}^{2+}$, and $\mathrm{O}^{+}$(see García-Rojas \& Esteban 2007). The origin of this discrepancy is still unknown and has been subject of debate for many years (see e.g., Tsamis \& Péquignot 2005; Stasińska et al. 2007; Mesa-Delgado et al. 2009b, 2012; Tsamis et al. 2011; Nicholls et al. 2012, 2013; Peimbert \& Peimbert 2013, and references therein for the most recent literature on the subject).

The comparison of nebular abundances with those resulting from the spectroscopic analysis of associated blue massive stars (especially B-type stars in the main sequence and BA supergiants) is another way to shed some light on the nebular abundance conundrum, particularly in the absence of nebular ORLs. These stars have been proved to be powerful alternative tools to derive the present-day chemical composition of the interstellar material in the Galactic regions where they are located (similarly to $\mathrm{H}$ II regions). Following this approach, several authors have compared galactic radial abundance gradients obtained from massive stars and $\mathrm{H}$ II regions in nearby spiral galaxies (see Bresolin et al. 2009; Trundle et al. 2002; U et al. 2009, for NGC 300, M 31, and M 33, respectively). When examined together, the outcome of these studies is, however, not completely conclusive. For example, while a total agreement between nebular and stellar abundances is observed in NGC 300 (Bresolin et al. 2009), a remarkable discrepancy is found in M 31 (Trundle et al. 2002). However, nebular and stellar abundances in most of these studies are compared in a general way, while there still exists the possibility of local or azimuthal variations of abundances in the studied galaxies that hamper a meaningful comparison of abundances. In addition, in several cases, nebular abundances could be only obtained by means of indirect (strong-line) methods and not directly from CELs/ORLs.

To avoid some of these limitations, one should concentrate on the study of $\mathrm{H}$ II regions and close-by blue massive stars for which we are confident that they have been formed and evolved in the same environment (and hence share the same chemical composition). In addition, a set of "technical" conditions must be taken into account: a) the H II region must be bright enough to detect the relatively faint auroral lines to determine $T_{\mathrm{e}}$ and, if possible, a set of ORLs; b) the spectra of the stars must show a statistically meaningful set of un-blended metallic lines (stars with spectral types in the range O9-B2 and low projected rotational velocities, $v \sin i<70 \mathrm{~km} \mathrm{~s}^{-1}$, are the most suitable targets for this study); c) stars must not belong to binary or multiple systems. There are not many Galactic H II regions that fulfill all these conditions. Of course, the keystone of all nebular stud-

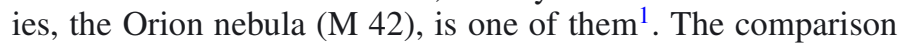
of nebular and stellar abundances in the Orion star-forming region (as derived from the analysis of the spectra of M 42 and a number of early B-type stars in the region ${ }^{2}$ ) has been recently reviewed by Simón-Díaz \& Stasińska (2011). The main conclusion of this study (which has been present in the literature for the past two decades, see e.g., Esteban et al. 1998, 2004) is that oxygen (gas+dust) nebular abundance based on ORLs agrees much better with the stellar abundances than the abundance derived

\footnotetext{
1 Of the few others we can cite M 16 (Eagle nebula), NGC 3372 (Carina nebula), or IC 5146 (Cocoon nebula, studied in this paper).

2 The nebular abundance analysis was performed by Esteban et al. (2004) and revisited by Simón-Díaz \& Stasińska (2011); the stellar abundance analysis was performed by Simón-Díaz (2010) and Nieva \& Simón-Díaz (2011).
}

from CELs. If a similarly thoughtful analysis of a larger sample of targets (including the analysis of other elements and considering different metallicity environments) were to confirm this result, this would have important implications for several fields of modern astrophysics and the way the abundance scale in the Universe is defined.

The Cocoon nebula (also known as IC 5146, Caldwell 19, and Sh 2-125) is an emission nebula located in the constelation of Cygnus. Its distance is somewhat uncertain and has been fixed between $950 \pm 80$ pc (Harvey et al. 2008) and $1200 \pm 180$ pc (Herbig \& Dahm 2002). This nebula is an ideal target to compare nebular and stellar abundances in detail in the same Galactic region since most of the technical conditions quoted above are fulfilled $^{3}$. The nebula is ionized by a single B0.5 V star BD463474, with a low projected rotational velocity - and is bright enough to compute physical conditions and chemical abundances from its nebular emission line spectrum. In addition, the ionization degree of the nebula is low because of the relatively low effective temperature of the central star, and therefore the most relevant elements are only single ionized and no ionization correction factors (ICF) are needed to determine total abundances of some key elements.

We perform a detailed spectroscopic abundance analysis of a set of long-slit spatially resolved intermediate-resolution spectra of the Cocoon nebula and a high-resolution optical spectrum of the ionizing star. The derived nebular CEL and stellar abundances of $\mathrm{O}, \mathrm{N}$, and $\mathrm{S}$ are then compared. The paper is structured as follows: the observational data set is presented in Sect. 2. The nebular physical conditions and abundances are determined in Sect. 3. A self-consistent spectroscopic abundance analysis of BD+463474 is performed in Sect. 4. The discussion of results and the main conclusions from our study are presented in Sects. 5 and 6.

\section{Observational data set}

\subsection{Nebular spectroscopy}

A long-slit, intermediate-resolution spectrum of the Cocoon nebula was obtained on 2011 November 23 with the Intermediate dispersion Spectrograph and Imaging System (ISIS) spectrograph attached to the $4.2 \mathrm{~m}$ William Herschel Telescope (WHT) at Roque de los Muchachos observatory in La Palma (Canary Islands, Spain). The $3.7^{\prime} \times 1.02^{\prime \prime}$ slit was located to the north-west of $\mathrm{BD}+463474,\left(\mathrm{PA}=320^{\circ}\right.$, see Fig. 1). Two different CCDs were used at the blue and red arms of the spectrograph: an EEV CCD with a configuration $4096 \times$ 2048 pixels at $13.5 \mu \mathrm{m}$, and a RedPlus CCD with $4096 \times$ 2048 pixels at $15.0 \mu \mathrm{m}$. The dichroic prism used to separate the blue and red beams was set at $5300 \AA$. The gratings R600B and $\mathrm{R} 316 \mathrm{R}$ were used for the blue and red observations. These gratings give a reciprocal dispersion of $33 \AA \mathrm{mm}^{-1}$ in the blue and $62 \AA \mathrm{mm}^{-1}$ in the red, effective spectral resolutions of 2.2 and $3.56 \AA$, and the spatial scales are $0.20^{\prime \prime}$ pixel $^{-1}$ and $0.22^{\prime \prime}$ pixel $^{-1}$. We used two different grating angles to cover the whole optical wavelength range: a) blue spectra centered on $4298 \AA$ and red spectra centered on $6147 \AA$; and b) blue spectra centered on $4298 \AA$ and red spectra centered on $8148 \AA$. The blue spectra cover an unvignetted range from $\lambda \lambda 3600$ to $5100 \AA$, the red range from $\lambda \lambda 5498$ to $9199 \AA$. The seeing during the observations was $\sim 2.0^{\prime \prime}$. The exposure times were $4 \times 1200 \mathrm{~s}$ in the

3 We did not detect ORLs in the spectrum of the Cocoon nebula, but the discussion can be held in other terms (see Sect. 5). 


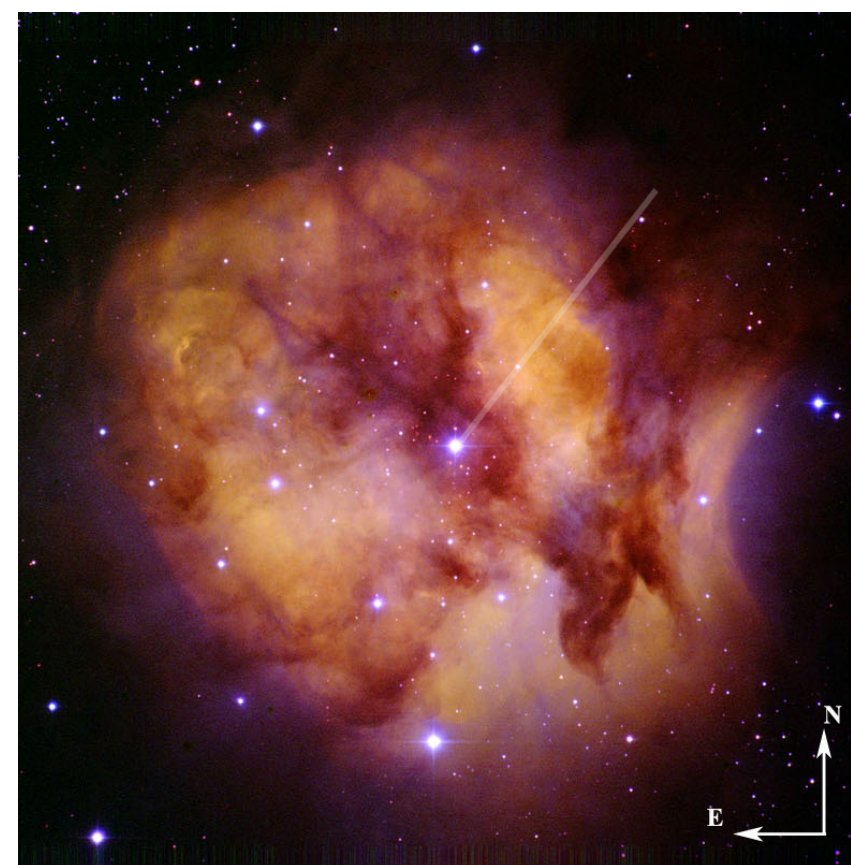

Fig. 1. Color-composite (RGB) image of the Cocoon nebula obtained from a combination of wide and narrow band images taken with the Wide Field Camera (WFC) at the $2.5 \mathrm{~m}$ Isaac Newton Telescope (only part of the CCD \#4 is shown) at the Roque de los Muchachos Observatory. The following color code was used: $\mathrm{H} \alpha$ (red), $\mathrm{H} \beta$ (green), B (blue) (credit of the image by Ángel R. López-Sánchez, Australian Astronomical Observatory). The field-of-view of the image is $\sim 11^{\prime} \times$ $11^{\prime}$. The position of the slit used to obtain the nebular ISIS at WHT spectroscopy is shown. The PA was $320^{\circ}$. Slit width is not to scale.

blue, $3 \times 1200 \mathrm{~s}$ in the red $\left(\lambda_{\mathrm{c}}=6147 \AA\right)$, and $1 \times 1200 \mathrm{~s}$ in the far red $\left(\lambda_{\mathrm{c}}=8148 \AA\right)$ observations.

The spectra were wavelength calibrated with a $\mathrm{CuNe}+\mathrm{CuAr}$ lamp. The correction for atmospheric extinction was performed using the average curve for continuous atmospheric extinction at Roque de los Muchachos Observatory. The absolute flux calibration was achieved by observations of the standard star $\mathrm{BD}+454655$. We used the IRAF ${ }^{4}$ TWODSPEC reduction package to perform bias correction, flat-fielding, cosmic-ray rejection, wavelength and flux calibration. We checked the relative flux calibration between the bluest and reddest wavelengths by calibrating the spectrophotometric standard by itself, finding a relative flux calibration uncertainty better than $\sim 5 \%$. Figure 2 shows an illustrative example of the quality of our nebular spectroscopic observations, where the main nebular lines used in this study are indicated. The sky emission could not be removed because the nebular emission was present along the whole slit.

\subsection{Stellar spectroscopy}

The spectroscopic observations ${ }^{5}$ of $\mathrm{BD}+463474$ were carried out with the FIES (Telting et al. 2014) cross-dispersed, high-resolution echelle spectrograph attached to the $2.56 \mathrm{~m}$ NOT telescope at El Roque de los Muchachos observatory on

\footnotetext{
4 IRAF is distributed by National Optical Astronomical Observatory which is operated by Association of Universities for Research in Astronomy Inc., under cooperative agreement with NSF.

5 The FIES spectrum of BD+463474 was obtained during one of the observing nights of the IACOB spectroscopic database of northern Galactic OB stars program (Simón-Díaz et al. 2011a).
}

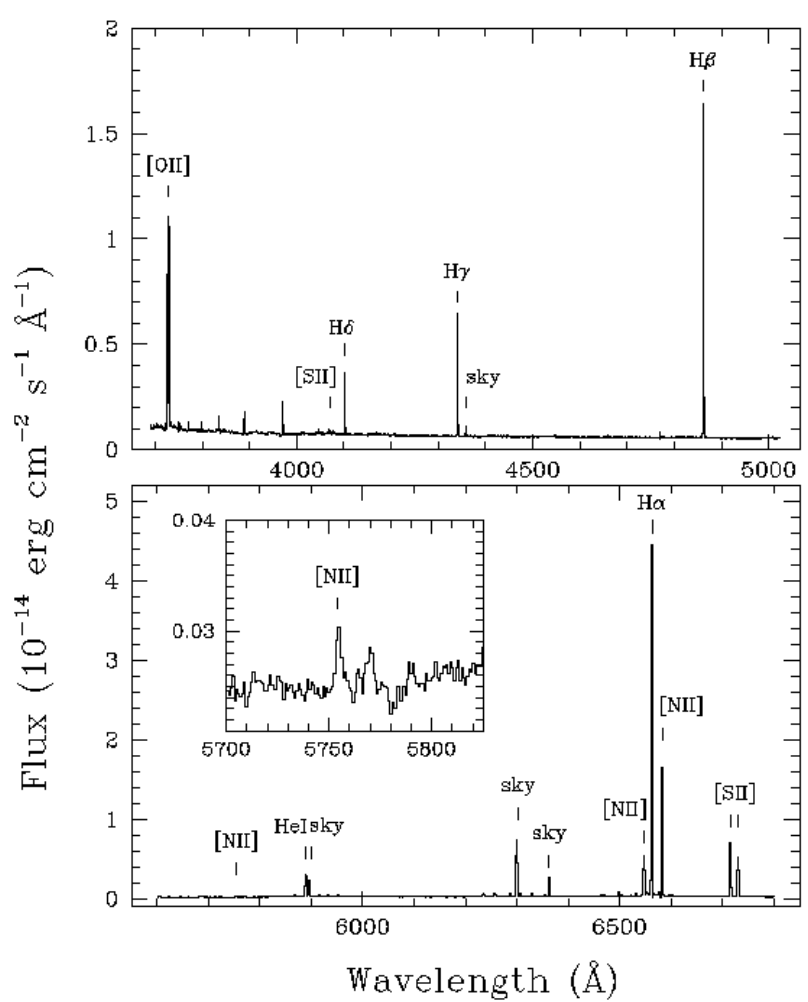

Fig. 2. Sections of the ISIS-WHT optical spectrum of the Cocoon nebula (aperture \#2) with some lines of interest labeled. The inset shows a zoom of the zone around [N II] $5755 \AA$. The mercury Hg I $4358 \AA$ line and other night-sky features are also indicated. Sky emission could not be removed from the spectrum (see text).

2012 September 10. The low-resolution mode $(R=25000$, $\delta \lambda=0.03 \AA /$ pix) was selected, and the entire spectral range 3700-7100 A was covered without gaps in a single fixed setting. We took one single spectrum with an exposure time of $1200 \mathrm{~s}$. The signal-to-noise ratio achieved was above 250 .

The spectrum was reduced with the FIEStool ${ }^{6}$ software in advanced mode. The FIEStool pipeline provided a wavelengthcalibrated, blaze-corrected, order-merged spectrum of high quality. This spectrum was then normalized with our own developed IDL routines. A selected range of the spectrum of BD+463474, where the main diagnostic lines used for the stellar parameter and abundance determination are indicated, is presented in Fig 3.

\section{Empirical analysis of the nebular spectra}

\subsection{Aperture selection and line flux measurements}

We obtained 1D spectra of zones of the nebula at different distances from the central star by dividing the long-slit used for the ISIS at WHT nebular observations in eight apertures within the limits of the nebula. Additionally, we extracted a spectrum containing all the apertures to compare it with the results obtained from the individual spectra.

The size of the apertures, except for the integrated one, was $22^{\prime \prime}$. The first aperture was centered $62.8^{\prime \prime}$ away from $\mathrm{BD}+463474$ to avoid the strong dust-scattered stellar continuum. A star about 78.3" away from BD+463474 was also avoided. The positions of the apertures are summarized in Table 1.

\footnotetext{
6 http://www .not.iac.es/instruments/fies/fiestool/ FIEStool.html
} 

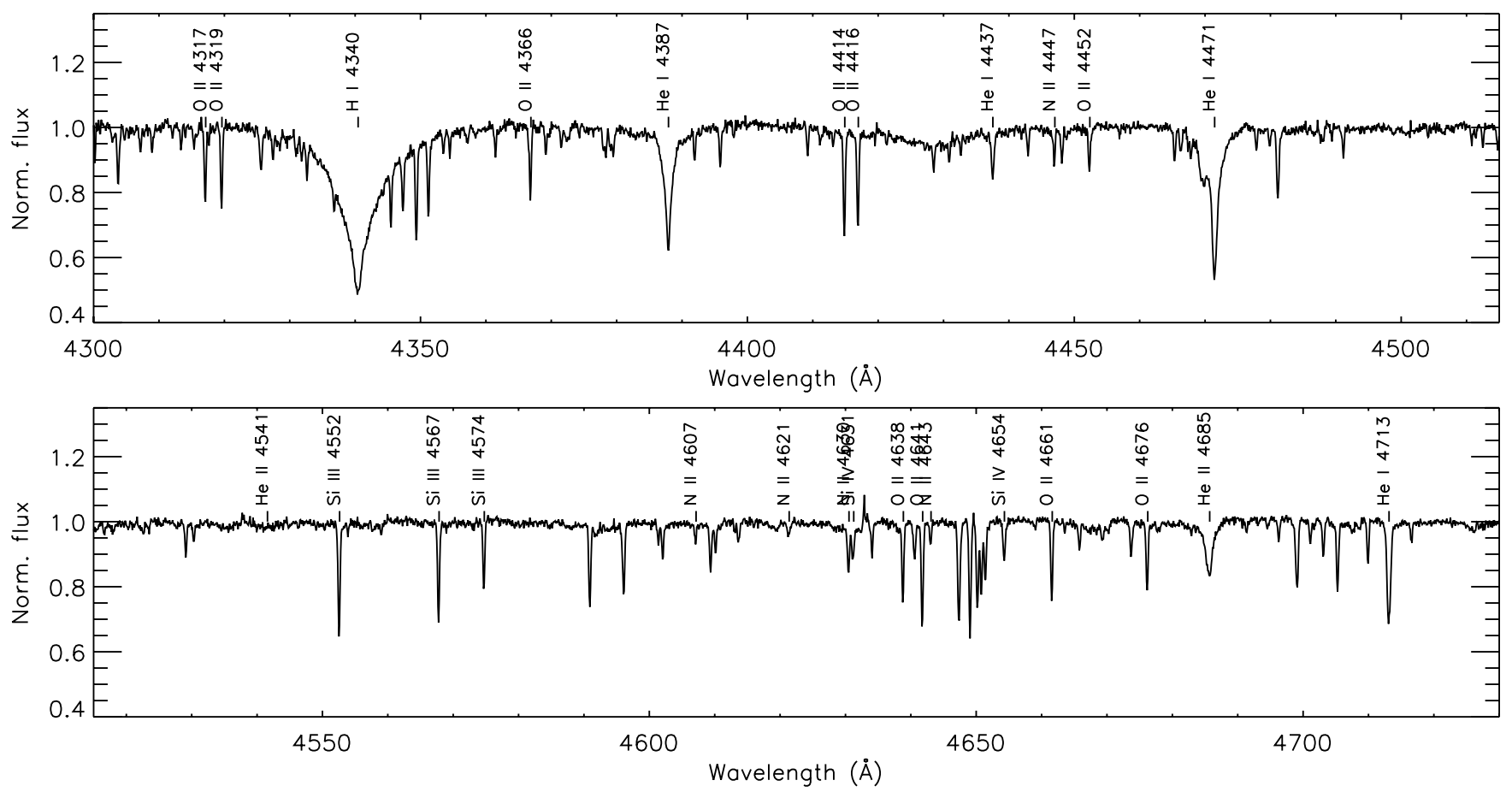

Fig. 3. Spectrum of BD+463474 in the region between 4300 and $4730 \AA$. In contrast to the broad hydrogen Balmer and He I lines $\left(\mathrm{H}_{\gamma}\right.$ and He I $\lambda \lambda$ 4387, 4471 and 4713 in the plotted spectral window), metal lines appear to be very narrow because of the low $v \sin i$ of the star. Only the [Si III]-IV, [O II], and [N II] lines present in the plotted region and used for the spectroscopic abundance analyis are indicated in the figure (see text and Table 4).

We extracted 1D spectra from each aperture with the IRAF task apall. The same zone and spatial coverage was considered in the blue and red spectroscopic ranges.

We detected HI and He I optical recombination lines, along with collisionally excited lines (CELs) of several low ionized ions, such as [O II], [N II], [S II], and [S III]. Line fluxes were measured using the SPLOT routine of the IRAF package by integrating all the flux included in the line profile between two given limits and over a local continuum estimated by eye.

Each emission line in the spectra was normalized to a particular H I recombination line present in each wavelength interval: $\mathrm{H} \beta$ for the blue range, $\mathrm{H} \alpha$ for the red spectra, and $\mathrm{P} 11$ for the far-red spectra.

The reddening coefficient $c(\mathrm{H} \beta)$ was obtained by fitting the observed $\mathrm{H} \delta / \mathrm{H} \beta$ and $\mathrm{H} \gamma / \mathrm{H} \beta$ line intensity ratios - the three lines lie in the same spectral range - to the theoretical ratios computed by Storey \& Hummer (1995) for $T_{\mathrm{e}}=6500 \mathrm{~K}$ and $n_{\mathrm{e}}=100 \mathrm{~cm}^{-3}$.

Finally, to produce a final homogeneous set of line intensity ratios, the red spectra were re-scaled to $\mathrm{H} \beta$ by applying the extinction correction and assuming the theoretical $\mathrm{H} \alpha / \mathrm{H} \beta$ and $\mathrm{P} 11 / \mathrm{H} \beta$ ratios: $I(\mathrm{H} \alpha) / I(\mathrm{H} \beta)=2.97$ and $I(\mathrm{P} 11) / I(\mathrm{H} \beta)=0.014$ obtained assuming $T_{\mathrm{e}}=6500 \mathrm{~K}$ and $n_{\mathrm{e}}=100 \mathrm{~cm}^{-3}$.

\subsection{Uncertainties}

Several sources of uncertainties must be taken into account to obtain the errors associated with the line intensity ratios. We estimated that the uncertainty in the line intensity measurement due to the signal-to-noise ratio of the spectra and the placement of the local continuum is typically $\sim 2 \%$ for $F(\lambda) / F(\mathrm{H} \beta) \geq 0.5$, $\sim 5 \%$ for $0.1 \leq F(\lambda) / F(\mathrm{H} \beta) \leq 0.5, \sim 10 \%$ for $0.05 \leq F(\lambda) / F(\mathrm{H} \beta)$ $\leq 0.1, \sim 20 \%$ for $0.01 \leq F(\lambda) / F(\mathrm{H} \beta) \leq 0.05, \sim 30 \%$ for $0.005 \leq$ $F(\lambda) / F(\mathrm{H} \beta) \leq 0.01$, and $\sim 40 \%$ for $0.001 \leq F(\lambda) / F(\mathrm{H} \beta) \leq 0.005$.
We did not consider lines weaker than $0.001 \times F(\mathrm{H} \beta)$. Note that the uncertainties indicated in Table 1 only refer to this type of errors.

By comparing the resulting flux-calibrated spectra of our standard star with the corresponding tabulated flux, we could estimate that line ratio uncertainties associated with the flux calibration is $\sim 3 \%$ when the wavelengths are separated by $500-1500 \AA$ and $\sim 5 \%$ if they are separated by more than that. For the cases where the corresponding lines are separated by less than $500 \AA$, the uncertainty in the line ratio due to uncertainties in the flux calibration is negligible.

The uncertainty associated with extinction correction was computed by error propagation. Again, the contribution of this uncertainty to the total error is negligible when line ratios of close-by lines are considered (e.g. [S II] $\lambda 6716 /[$ S II] $\lambda 6730$ ). The final errors in the line intensity ratios used to derive the physical properties of the nebula were computed by adding quadratically these three sources of uncertainty.

\subsection{Physical conditions}

Electron temperature $\left(T_{\mathrm{e}}\right)$, and density $\left(n_{\mathrm{e}}\right)$ of the ionized gas were derived from classical CEL ratios, using the pyhton-based code PyNeb (Luridiana et al. 2014) and the set of atomic data shown in Table 1 . We computed $n_{\mathrm{e}}$ from the [S II] $\lambda 6717 / \lambda 6731$ and [O II] $\lambda 3729 / \lambda 3726$ line ratios and $T_{\mathrm{e}}$ from the nebular to auroral [N II] $\lambda \lambda(6548+84) / \lambda 5754$ line ratio and from [S II] $\lambda \lambda(6717+31) / \lambda \lambda(4068+76)$.

The methodology used for determining the physical conditions was as follows: we assumed a representative initial value of $T_{\mathrm{e}}$ of $10000 \mathrm{~K}$ and computed the electron densities. Then, the value of $n_{\mathrm{e}}$ was used to compute $T_{\mathrm{e}}([\mathrm{N} \mathrm{II}])$ and/or $T_{\mathrm{e}}([\mathrm{S} \mathrm{II}])$ from the observed line ratios; for the four innermost apertures, 
Table 1. Line fluxes ${ }^{1}$ corrected for extinction $(\mathrm{H} \beta=100)$, and results from the empirical analysis ${ }^{2}$ for CELs of the nebular spectra extrated from the 8 apertures and the integrated spectrum.

\begin{tabular}{|c|c|c|c|c|c|c|c|c|c|c|c|}
\hline & & & \multicolumn{9}{|c|}{ Aperture } \\
\hline & & & $\mathrm{A} 1$ & $\mathrm{~A} 2$ & A3 & $\mathrm{A} 4$ & A5 & A6 & A7 & A8 & Integrated \\
\hline \multirow{2}{*}{\multicolumn{3}{|c|}{$\begin{array}{r}\text { Center position }{ }^{3}(\operatorname{arcsec}) \\
\text { Angular area }\left(\operatorname{arcsec}^{2}\right)\end{array}$}} & 62.8 & 93.8 & 115.8 & 137.8 & 159.8 & 181.8 & 203.8 & 225.8 & 144.3 \\
\hline & & & 22 & 22 & 22 & 22 & 22 & 22 & 22 & 22 & 176 \\
\hline$\lambda(\AA)$ & Ion & Mult. & \multicolumn{9}{|c|}{$I(\lambda) / I(\mathrm{H} \beta)$} \\
\hline 3726.03 & [O II] & $1 \mathrm{~F}$ & $74 \pm 6$ & $72 \pm 2$ & $73 \pm 3$ & $80 \pm 2$ & $94 \pm 5$ & $93 \pm 7$ & $107 \pm 5$ & $129 \pm 18$ & $80 \pm 4$ \\
\hline 3728.82 & [O II] & $1 \mathrm{~F}$ & $103 \pm 7$ & $102 \pm 3$ & $102 \pm 3$ & $112 \pm 3$ & $130 \pm 7$ & $129 \pm 9$ & $149 \pm 6$ & $172 \pm 22$ & $111 \pm 5$ \\
\hline 4068.60 & {$[\mathrm{~S}$ II] } & $1 \mathrm{~F}$ & $2.5 \pm 0.5$ & $2.6 \pm 0.3$ & $2.8 \pm 0.5$ & $3.1 \pm 0.4$ & $3.2 \pm 0.3$ & $2.8 \pm 0.6$ & $6 \pm 1$ & - & $2.8 \pm 0.4$ \\
\hline 4076.35 & [S II] & $1 \mathrm{~F}$ & $1.4 \pm 0.4$ & $1.6 \pm 0.2$ & $1.7 \pm 0.2$ & $1.9 \pm 0.3$ & $3.9 \pm 0.7$ & $4 \pm 1$ & $6 \pm 2$ & - & $2.3 \pm 0.3$ \\
\hline 4101.74 & H I & $\mathrm{H} \delta$ & $25 \pm 1$ & $25.3 \pm 0.7$ & $25.8 \pm 0.7$ & $25.6 \pm 0.7$ & $26 \pm 2$ & $27 \pm 2$ & $26 \pm 2$ & $24 \pm 5$ & $26 \pm 1$ \\
\hline 4340.47 & $\mathrm{HI}$ & $\mathrm{H} \gamma$ & $47 \pm 2$ & $46 \pm 1$ & $46 \pm 1$ & $46 \pm 1$ & $46 \pm 2$ & $46 \pm 2$ & $46 \pm 2$ & $47 \pm 7$ & $46 \pm 1$ \\
\hline 4861.33 & H I & $\mathrm{H} \beta$ & $100 \pm 2$ & $100 \pm 2$ & $100 \pm 2$ & $100 \pm 2$ & $100 \pm 2$ & $100 \pm 2$ & $100 \pm 2$ & $100 \pm 2$ & $100 \pm 2$ \\
\hline 5754.64 & {$[\mathrm{~N}$ II] } & $3 \mathrm{~F}$ & $0.51 \pm 0.07$ & $0.53 \pm 0.05$ & $0.62 \pm 0.08$ & $0.6 \pm 0.1$ & - & - & - & - & $0.5 \pm 0.1$ \\
\hline 5875.64 & $\mathrm{He} \mathrm{I}$ & 11 & $2.1 \pm 0.2$ & - & - & - & - & - & - & - & $1.0 \pm 0.2$ \\
\hline 6548.03 & {$[\mathrm{~N}$ II $]$} & $1 \mathrm{~F}$ & $36 \pm 2$ & $35 \pm 1$ & $38 \pm 1$ & $37 \pm 1$ & $39 \pm 3$ & $41 \pm 2$ & $39 \pm 1$ & $42 \pm 4$ & $37 \pm 2$ \\
\hline 6562.82 & H I & $\mathrm{H} \alpha$ & $297 \pm 18$ & $297 \pm 8$ & $297 \pm 8$ & $297 \pm 8$ & $297 \pm 20$ & $297 \pm 15$ & $297 \pm 6$ & $297 \pm 23$ & $297 \pm 15$ \\
\hline 6583.41 & {$[\mathrm{~N} \mathrm{II}]$} & $1 \mathrm{~F}$ & $111 \pm 7$ & $108 \pm 3$ & $109 \pm 3$ & $113 \pm 3$ & $115 \pm 8$ & $122 \pm 6$ & $124 \pm 3$ & $121 \pm 9$ & $111 \pm 6$ \\
\hline 6678.15 & $\mathrm{He} \mathrm{I}$ & 46 & $0.44 \pm 0.06$ & - & - & - & - & - & - & - & - \\
\hline 6716.47 & {$[\mathrm{~S} \mathrm{II}]$} & $2 \mathrm{~F}$ & $41 \pm 3$ & $45 \pm 1$ & $51 \pm 2$ & $59 \pm 2$ & $61 \pm 4$ & $67 \pm 4$ & $72 \pm 2$ & $71 \pm 6$ & $51 \pm 3$ \\
\hline 6730.85 & {$[\mathrm{~S} \mathrm{II}]$} & $2 \mathrm{~F}$ & $30 \pm 2$ & $33.2 \pm 0.9$ & $38 \pm 1$ & $43 \pm 1$ & $44 \pm 3$ & $49 \pm 3$ & $52 \pm 1$ & $50 \pm 4$ & $37 \pm 2$ \\
\hline 8862.79 & $\mathrm{HI}$ & P11 & $1.4 \pm 0.2$ & $1.4 \pm 0.1$ & $1.4 \pm 0.1$ & $1.4 \pm 0.2$ & $1.4 \pm 0.5$ & $1.4 \pm 0.4$ & $1.4 \pm 0.3$ & 1.4: & $1.4 \pm 0.2$ \\
\hline 9014.91 & H I & P10 & $1.1 \pm 0.3$ & $1.2 \pm 0.1$ & $1.2 \pm 0.1$ & $1.1 \pm 0.2$ & 1.0: & $0.6:$ & $0.4:$ & - & $1.1 \pm 0.3$ \\
\hline \multirow[t]{14}{*}{9068.90} & [S III] & $1 \mathrm{~F}$ & $6 \pm 1$ & $6.5 \pm 0.4$ & $4.5 \pm 0.3$ & $3.5 \pm 0.3$ & $2.9 \pm 0.8$ & $2.5 \pm 0.8$ & $1.9 \pm 0.6$ & 1.7: & $4.4 \pm 0.6$ \\
\hline & & $c(\mathrm{H} \beta)$ & $1.07 \pm 0.10$ & $0.79 \pm 0.02$ & $0.89 \pm 0.04$ & $0.61 \pm 0.02$ & $0.90 \pm 0.08$ & $0.95 \pm 0.11$ & $1.12 \pm 0.04$ & $1.65 \pm 0.21$ & $0.88 \pm 0.06$ \\
\hline & & $F(\mathrm{H} \beta)^{4}$ & $2.39 \pm 0.05$ & $2.72 \pm 0.05$ & $2.49 \pm 0.05$ & $2.07 \pm 0.04$ & $0.78 \pm 0.02$ & $0.74 \pm 0.02$ & $0.40 \pm 0.01$ & $0.26 \pm 0.01$ & $11.8 \pm 0.2$ \\
\hline & & $n_{\mathrm{e}}([\mathrm{O} \quad \mathrm{II}])$ & $63_{-63}^{+59}$ & $50 \pm 28$ & $56 \pm 35$ & $53 \pm 25$ & $59_{-59}^{+106}$ & $58_{-58}^{+76}$ & $41 \pm 29$ & $28_{-28}^{+110}$ & $60 \pm 43$ \\
\hline & & $n_{\mathrm{e}}([\mathrm{S} \mathrm{II}])$ & $48_{-48}^{-68}$ & $50 \pm 30$ & $50 \pm 34$ & $50 \pm 31$ & $61 \pm 56$ & $63_{-63}^{-69}$ & $63 \pm 46$ & $90_{-90}^{+149}$ & $46_{-46}^{+60}$ \\
\hline & & $T_{\mathrm{e}}([\mathrm{N} \mathrm{II}])$ & $6850 \pm 280$ & $7020 \pm 210$ & $7220 \pm 280$ & $7230 \pm 420$ & - & -63 & - & -90 & $6900 \pm 490$ \\
\hline & & $T_{\mathrm{e}}([\mathrm{S} \mathrm{II}])$ & $8190 \pm 810$ & $8030 \pm 430$ & $7700 \pm 520$ & $7650 \pm 390$ & $9360 \pm 820$ & $8800 \pm 1000$ & $12000 \pm 2500$ & - & $8410 \pm 580$ \\
\hline & & $\mathrm{O}^{+} / \mathrm{H}^{+}$ & $8.63 \pm 0.06$ & $8.55 \pm 0.05$ & $8.48 \pm 0.06$ & $8.52 \pm 0.10$ & - & - & - & - & $8.64 \pm 0.13$ \\
\hline & & $\mathrm{N}^{+} / \mathrm{H}^{+}$ & $7.87 \pm 0.07$ & $7.82 \pm 0.04$ & $7.79 \pm 0.05$ & $7.79 \pm 0.07$ & - & - & - & - & $7.86 \pm 0.10$ \\
\hline & & $\mathrm{S}^{+} / \mathrm{H}^{+}$ & $6.69 \pm 0.07$ & $6.69 \pm 0.04$ & $6.71 \pm 0.05$ & $6.76 \pm 0.07$ & - & - & - & - & $6.77 \pm 0.10$ \\
\hline & & $\mathrm{S}^{2+} / \mathrm{H}^{+}$ & $6.30 \pm 0.12$ & $6.17 \pm 0.07$ & $6.10 \pm 0.08$ & $5.99 \pm 0.11$ & - & - & - & - & $6.15 \pm 0.15$ \\
\hline & & $\mathrm{O} / \mathrm{H}$ & $8.63 \pm 0.06$ & $8.55 \pm 0.05$ & $8.48 \pm 0.06$ & $8.52 \pm 0.10$ & - & - & - & - & $8.64 \pm 0.13$ \\
\hline & & $\mathrm{N} / \mathrm{H}$ & $7.87 \pm 0.07$ & $7.82 \pm 0.04$ & $7.79 \pm 0.05$ & $7.79 \pm 0.07$ & - & - & - & - & $7.86 \pm 0.10$ \\
\hline & & $\mathrm{S} / \mathrm{H}$ & $6.84 \pm 0.06$ & $6.81 \pm 0.04$ & $6.80 \pm 0.04$ & $6.83 \pm 0.06$ & - & - & - & - & $6.86 \pm 0.08$ \\
\hline
\end{tabular}

Notes. ${ }^{(1)}$ The errors in the line fluxes only refer to uncertainties in the line measurements (see text). (2) $n_{\mathrm{e}}$ in $\mathrm{cm}^{-3} ; T_{\mathrm{e}}$ in $\mathrm{K}$; abundances in $\log \left(\mathrm{X}^{+i} / \mathrm{H}^{+}\right)+12 .{ }^{(3)}$ With respect to $\mathrm{BD}+463474 .{ }^{(4)} \mathrm{F}(\mathrm{H} \beta)$ in $\times 10^{-14} \mathrm{erg} \mathrm{cm}^{2} \mathrm{~s}^{-1}$ and uncorrected for reddening.

we only assumed $T_{\mathrm{e}}([\mathrm{N}$ II $]$ ) to be valid (see below). We iterated until convergence to obtain the final values of $n_{\mathrm{e}}$ and $T_{\mathrm{e}}$. Uncertainties were computed by error propagation. The final $n_{\mathrm{e}}([\mathrm{O} \mathrm{II}]), n_{\mathrm{e}}([\mathrm{S} \mathrm{II}]), T_{\mathrm{e}}([\mathrm{N}$ II $])$ and $T_{\mathrm{e}}([\mathrm{S}$ II $])$ estimate along with their uncertainties are indicated in Table 1 . We did not rely on the determination of $T_{\mathrm{e}}$ ([S II]) for apertures $5-7$, because it is much higher than the $T_{\mathrm{e}}$ obtained in the other apertures, and yield unreasonably low values for the chemical abundances of $\mathrm{O}$, $\mathrm{N}$, and $\mathrm{S}$.

In general, densities derived from the [O II] line ratio are very consistent with those derived from [S II] lines for all the apertures, and aditionally, they are also homogeneous for all the apertures. On the other hand, the temperatures derived from the [N II] and [S II] line ratios present considerable differences for a given aperture, especially in the inner aperture and in the total extraction. Given the high dependence of the value of $T_{\mathrm{e}}([\mathrm{S} \mathrm{II}])$ on the set of collisional strengths adopted and that the $\mathrm{S}^{+}$zone does not entirely match the $\mathrm{N}^{+}$zone $\left(\mathrm{S}^{+}\right.$is in the outer zones of the nebula, whereas $\mathrm{N}^{+}$is probably more evenly distributed along the nebula), we decided to only adopt $T_{\mathrm{e}}([\mathrm{N} \mathrm{II}])$ as representative of the whole nebula.

\subsection{Chemical abundances}

We have detected only two He I lines in the innermost aperture. Because the hydrogen lines on this aperture could be affected by fluorescense effects (see below), we decided against computing the $\mathrm{He}^{+}$abundance that should be, in any case, a very low limit of the total He abundance because of the low ionization degree of the nebula.

Ionic abundances of $\mathrm{N}^{+}, \mathrm{O}^{+}, \mathrm{S}^{+}$, and $\mathrm{S}^{2+}$ were derived from CELs with PyNeb. We assumed a one-zone scheme, in which we adopted $T_{\mathrm{e}}([\mathrm{N}$ II $])$ to compute the $\mathrm{N}^{+}, \mathrm{O}^{+}, \mathrm{S}^{+}$and $\mathrm{S}^{2+}$ abundances. We assumed the average of $n_{\mathrm{e}}([\mathrm{S}$ II $])$ and $n_{\mathrm{e}}([\mathrm{O} \mathrm{II}])$ as representative for each aperture. Owing to the low $T_{\text {eff }}$ of the ionizing star, we did not detect CELs of high-ionization species, such as $\mathrm{O}^{2+}, \mathrm{Ar}^{2+}$ or $\mathrm{Ne}^{2+}$. For the same reason, O II ORLs were not detected in our spectrum. Additionally, the O I ORLs in the 7771-7775 A range were not detected either in our spectrum because they are intrinsically faint and lie in a zone with strong sky emission; therefore, we could not compute the abundance discrepancies for $\mathrm{O}^{+}$or for $\mathrm{O}^{++}$in this nebula (see Sect. 1).

We then derived total abundances of $\mathrm{O}, \mathrm{N}$, and $\mathrm{S}$ for each aperture without using ICFs. We stress that this is important because one of the main uncertainty sources in total abundances determinations in $\mathrm{H}$ II regions come from the necessity of using ICFs (see more details in Sect. 5). The ionic abundances of $\mathrm{O}^{+}$, $\mathrm{N}^{+}, \mathrm{S}^{+}, \mathrm{S}^{2+}$ along with the total abundances of $\mathrm{O}, \mathrm{N}$, and $\mathrm{S}$ are shown in Table 1.

From Table 1 it can be seen that both physical conditions and total chemical abundances obtained from the innermost and from the integrated aperture are somewhat different from those obtained from apertures 2, 3, and 4, which are remarkably consistent. In principle, one should not expect a variation in 
the total abundance obtained for a given element, especially if they were derived directly from ionic abundances, without using any ICF. Ferland (1999) and Luridiana et al. (2009) described the importance of fluorescent excitation of Balmer lines due to continuum pumping in the hydrogen Lyman transitions by nonionizing stellar continua. In particular, Luridiana et al. (2009) described this effect and its behaviour with spectral type, luminosity class, and distance to the star in detail for enviroments where Lyman transitions are optically thick. In our case, this effect can be particularly important in aperture 1, which is the closest to the star. This effect depends on several parameters and can only be adressed with a detailed photoionization model with an appropiate high-resolution sampling of the non-ionizing spectrum of the stellar source (Luridiana et al. 2009). From a qualitative approach, differential fluorescent excitation of $\mathrm{H}$ I lines can mainly affect the determination of the extinction coefficient, $c(\mathrm{H} \beta)$, which can be overestimated in the apertures closest to the ionizing star (Simón-Díaz et al. 2011b, reported this effect in the inner apertures of a long-slit study of M 43); the overall effect would be i) an overestimation of the line fluxes bluer than $\mathrm{H} \beta$ in the blue range; ii) an underestimation of line fluxes in the far-red range; and iii) an overestimation or underestimation of fluxes for lines bluer or redder than $\mathrm{H} \alpha$ in the red range. This effect would only substantially affect the $\mathrm{O}^{+} / \mathrm{H}^{+}$abundances because [O II] line fluxes would be overestimated. The effect in $\mathrm{N}^{+}$would be very small, because of the proximity of these lines to $\mathrm{H} \alpha$. Sulphur ionic abundances are affected by ionization structure effects and cannot be discussed on these terms. On the other hand, the effect in the determination of $n_{\mathrm{e}}$ would be negligible because both $n_{\mathrm{e}}([\mathrm{O} \mathrm{II}])$ and $n_{\mathrm{e}}([\mathrm{S} \mathrm{II}])$ are computed from line ratios belonging to the same range and very close in wavelength; finally, a small effect would emerge in the determination of $T_{\mathrm{e}}([\mathrm{N} \mathrm{II}])$ mainly because of the difference in wavelength between auroral and nebular [N II] lines. Taking into account all these possible effects, we do not consider aperture 1 computations because of the remarkable differences with apertures 2, 3, and 4, and we consider the weighted average of these three apertures as representative of the whole nebula.

In last column of Table 1 we also present the results for the integrated spectra, for comparative purposes. The results obtained for the integrated spectra are very similar to those obtained in aperture 1. This is probably due to the effect of apertures 5 to 8 in the collapsed spectrum; these four apertures show higher values of $c(\mathrm{H} \beta)$ than apertures 2 to 4 , hence mimic the effect observed in aperture 1 .

\section{Quantitative spectroscopic analysis of BD+46 3474}

We followed a similar strategy as described in Simón-Díaz (2010) to perform a detailed, self-consistent spectroscopic abundance analysis of BD+463474 by means of the modern stellar atmosphere code FASTWIND (Santolaya-Rey et al. 1997; Puls et al. 2005). In brief, the stellar parameters were derived by comparing the observed $\mathrm{H}$ Balmer line profiles and the ratio of Si III-IV line equivalent widths (EWs) with the output from a grid of FASTWIND models. Then, the same grid of models was used to derive the stellar abundances by means of the curve-ofgrowth method.

The projected rotational velocity $(v \sin i)$ of the star was derived by means of the iacob-broad procedure implemented in IDL (see notes about its performance in Simón-Díaz \& Herrero 2014) and the measurement of the equivalenth width of the
Table 2. Atomic data considered in the nebular abundance analysis.

\begin{tabular}{lll}
\hline \hline & & CELs \\
Ion & Trans. probabilities & Coll. strengths \\
\hline $\mathrm{N}^{+}$ & Galavis et al. (1997) & Tayal (2011) \\
$\mathrm{O}^{+}$ & Zeippen (1982) & Pradhan et al. (2006) \\
$\mathrm{S}^{+}$ & Podobedova et al. (2009) & Ramsbottom et al. (1996) \\
$\mathrm{S}^{2+}$ & Podobedova et al. (2009) & Galavís et al. (1995) \\
\hline
\end{tabular}

Table 3. Spectroscopic parameters and abundances of $\mathrm{Si}, \mathrm{O}$, and $\mathrm{N}$ derived through the FASTWIND analysis of the optical spectrum of $\mathrm{BD}+463474$.

\begin{tabular}{lc|lc}
\hline \hline$T_{\text {eff }}(\mathrm{K})$ & $30500 \pm 1000$ & $V$ & 9.74 \\
$\log g(\mathrm{dex})$ & $4.2 \pm 0.1$ & $(B-V)$ & 0.78 \\
$Y(\mathrm{He})$ & $0.11 \pm 0.02$ & $(B-V)_{0}$ & -0.27 \\
$\log \mathrm{Q}$ & $<-13.5$ & $E(B-V)$ & 1.05 \\
$\zeta_{\mathrm{t}}\left(\mathrm{km} \mathrm{s}^{-1}\right)$ & $<5$ & $A_{V}$ & 3.25 \\
$v \sin i\left(\mathrm{~km} \mathrm{~s}^{-1}\right)$ & $<15$ & $M_{V}$ & $-3.0^{1}$ \\
$\log (\mathrm{Si} / \mathrm{H})+12$ & $7.51 \pm 0.05$ & $R\left(R_{\odot}\right)$ & $5.2 \pm 0.2^{1}$ \\
$\log (\mathrm{O} / \mathrm{H})+12$ & $8.73 \pm 0.08$ & $\log L / L_{\odot}$ & $4.29 \pm 0.03^{1}$ \\
$\log (\mathrm{N} / \mathrm{H})+12$ & $7.86 \pm 0.05$ & $M_{\mathrm{sp}}\left(M_{\odot}\right)$ & $15 \pm 2^{1}$ \\
\hline
\end{tabular}

Notes. (1) Corresponding to a distance of $800 \mathrm{pc}$ (see notes in Appendix A).

metal lines of interest was performed as described in SimónDíaz (2010). While Simón-Díaz (2010) concentrated on the oxygen and silicon abundance determination, in the present study we were able to also extend the abundance analysis to nitrogen, taking advantage of the implementation of a new nitrogen model atom into the FASTWIND code (Rivero González et al. 2011, 2012).

In a first step we used the $\mathrm{H}_{\gamma}$ line, along with the ratio EW(Si IV 24116)/EW(Si III 24552) to constrain the effective temperature and gravity, obtaining $30100 \pm 500,30500 \pm 500$, and $30800 \pm 700 \mathrm{~K}$ for $\log g=4.1,4.2$, and $4.3 \mathrm{dex}$, respectively (with $\log g=4.2$ dex providing the best by-eye fit to $\mathrm{H} \gamma$ ). An independent determination of these two parameters, together with the helium abundance $\left(Y_{\mathrm{He}}\right)$, the microturbulence $\left(\zeta_{\mathrm{t}}\right)$, and the wind-strength $Q$-parameter $\left(\dot{M}\left(R v_{\infty}\right)^{-3 / 2}\right.$, Puls et al. 1996) was obtained by performing a HHe spectroscopic analysis using the iacob-gbat package (Simón-Díaz et al. 2011a). In this case we used the full set of $\mathrm{H}$ and $\mathrm{He}$ I-II lines available in the FIES spectrum, obtaining a perfect agreement with the results from the HSi analysis $\left(T_{\text {eff }}=30100 \pm 1000 \mathrm{~K}\right.$ and $\left.\log g=4.2 \pm 0.1 \mathrm{dex}\right)$, plus $Y_{\mathrm{He}}=0.11 \pm 0.02, \zeta_{\mathrm{t}}<5 \mathrm{~km} \mathrm{~s}^{-1}$, and $\log Q<-13.5$.

The final set of stellar parameters derived spectroscopically (along with the corresponding uncertainties) is summarized in Table 3. For completeness, we also include in Table 3 the derived $\mathrm{Si}, \mathrm{O}$, and $\mathrm{N}$ abundances (see below) as well as other stellar parameters of interest for further studies of the Cocoon nebula and its ionizing source such as the radius, luminosity, and spectroscopic mass (see notes about how these parameters were derived in Appendix A). The spectroscopic parameters were then fixed for the subsequent $\mathrm{Si}, \mathrm{O}$, and $\mathrm{N}$ abundance analysis. We present in Table 4 the considered set of Si III-IV, O II, and N II-III diagnostic lines, along with the measured EWs and corresponding line-by-line abundances ${ }^{7}$ (plus the associated uncertainties). The same information is represented graphically in Fig. 4.

$\epsilon_{\mathrm{X}}=\log (X / \mathrm{H})+12$ 
J. García-Rojas et al.: The Cocoon nebula and its ionizing star

Table 4. Results from the abundance analysis of BD +463474 (B0.5 V).

\begin{tabular}{|c|c|c|c|c|}
\hline \multirow{2}{*}{$\frac{\mathrm{BD}+463474}{\text { Line }}$} & \multicolumn{3}{|c|}{$T_{\text {eff }}=30500 \mathrm{~K}, \log g=4.2 \mathrm{dex}$} & \multirow{2}{*}{$\begin{array}{c}\zeta_{\mathrm{t}}(\mathrm{Si})=1 \\
\Delta \epsilon_{\mathrm{Si}} \\
(\mathrm{dex})\end{array}$} \\
\hline & $\begin{array}{c}E W \\
(\mathrm{~m} \AA)\end{array}$ & $\begin{array}{l}\Delta E W \\
(\mathrm{~m} \AA)\end{array}$ & $\begin{array}{c}\epsilon_{\mathrm{Si}} \\
(\mathrm{dex})\end{array}$ & \\
\hline Si III $\lambda 4552$ & 127 & 5 & 7.48 & 0.09 \\
\hline Si III $\lambda 4567$ & 111 & 5 & 7.51 & 0.09 \\
\hline Si III $\lambda 4574$ & 73 & 4 & 7.53 & 0.09 \\
\hline Si IV $\lambda 4116$ & 106 & 10 & 7.48 & 0.18 \\
\hline Si IV $\lambda 4212$ & 33 & 4 & 7.58 & 0.14 \\
\hline Si IV $\lambda 4631$ & 50 & 10 & 7.52 & 0.23 \\
\hline Si IV $\lambda 4654$ & 51 & 12 & 7.28 & 0.29 \\
\hline Si IV $\lambda 6667$ & 13 & 3 & 7.43 & 0.20 \\
\hline \multirow[t]{2}{*}{ Si IV $\lambda 6701$} & 25 & 5 & 7.54 & 0.20 \\
\hline & \multicolumn{2}{|c|}{$\Delta \zeta_{\mathrm{t}}(\mathrm{Si})=1$} & $\begin{array}{c}\epsilon_{\mathrm{Si}}=7.51 \\
\Rightarrow\end{array}$ & $\begin{aligned} \Delta \epsilon_{\mathrm{Si}}(\sigma) & =0.05 \\
\Delta \epsilon_{\mathrm{Si}}\left(\zeta_{\mathrm{t}}\right) & =0.06\end{aligned}$ \\
\hline $\mathrm{BD}+463474$ & \multicolumn{3}{|c|}{$T_{\mathrm{eff}}=30500 \mathrm{~K}, \log g=4.2 \mathrm{dex}$} & $\zeta_{\mathrm{t}}(\mathrm{O})=1$ \\
\hline О ІІ $\lambda 3945$ & 54 & 9 & 8.86 & 0.22 \\
\hline О II $\lambda 3954$ & 77 & 8 & 8.83 & 0.17 \\
\hline О ІІ $\lambda 4317$ & 102 & 12 & 8.78 & 0.17 \\
\hline О ІІ $\lambda 4319$ & 87 & 6 & 8.70 & 0.11 \\
\hline О ІІ $\lambda 4366$ & 82 & 8 & 8.66 & 0.16 \\
\hline О ІІ $\lambda 4414$ & 118 & 6 & 8.66 & 0.10 \\
\hline О ІІ $\lambda 4416$ & 109 & 6 & 8.85 & 0.11 \\
\hline О ІІ $\lambda 4452$ & 52 & 8 & 8.80 & 0.19 \\
\hline O ІІ $\lambda 4638$ & 88 & 7 & 8.98 & 0.16 \\
\hline О ІІ $\lambda 4641$ & 91 & 3 & 8.50 & 0.06 \\
\hline О ІІ $\lambda 4661$ & 87 & 5 & 8.80 & 0.11 \\
\hline О ІІ $\lambda 4676$ & 82 & 5 & 8.80 & 0.11 \\
\hline О ІІ $\lambda 6721$ & 51 & 3 & 8.74 & 0.06 \\
\hline О ІІ $\lambda 4076$ & 108 & 11 & 8.70 & 0.24 \\
\hline О ІІ $\lambda 4891$ & 27 & 4 & 8.65 & 0.14 \\
\hline О ІІ $\lambda 4906$ & 45 & 6 & 8.69 & 0.16 \\
\hline О II $\lambda 4941$ & 43 & 4 & 8.58 & 0.10 \\
\hline \multirow[t]{2}{*}{ O II $\lambda 4943$} & 63 & 3 & 8.65 & 0.07 \\
\hline & \multicolumn{2}{|c|}{$\Delta \zeta_{\mathrm{t}}(\mathrm{O})=1$} & $\begin{aligned} \epsilon_{\mathrm{O}} & =\mathbf{8 . 7 3} \\
& \Rightarrow \\
& \Rightarrow\end{aligned}$ & $\begin{aligned} & \Delta \epsilon_{\mathrm{O}}(\sigma)=0.08 \\
& \Delta \epsilon_{\mathrm{O}}\left(\zeta_{\mathrm{t}}\right)=0.03 \\
& \Delta \epsilon_{\mathrm{O}}(\mathrm{SP})=0.08 \\
&\end{aligned}$ \\
\hline $\mathrm{BD}+463474$ & \multicolumn{3}{|c|}{$T_{\mathrm{eff}}=30500 \mathrm{~K}, \log g=4.2 \mathrm{dex}$} & $\zeta_{\mathrm{t}}(\mathrm{N})=1$ \\
\hline N II $\lambda 3995$ & 81 & 10 & 8.11 & 0.16 \\
\hline N II $\lambda 4607$ & 20 & 10 & 7.86 & 0.45 \\
\hline N II $\lambda 4621$ & 20 & 7 & 7.86 & 0.30 \\
\hline N II $\lambda 4630$ & 55 & 15 & 7.89 & 0.30 \\
\hline N II $\lambda 4643$ & 25 & 2 & 7.81 & 0.07 \\
\hline N II $\lambda 4447$ & 38 & 8 & 7.92 & 0.20 \\
\hline N II $\lambda 5045$ & 28 & 10 & 7.93 & 0.33 \\
\hline N II $\lambda 5666$ & 34 & 5 & 7.77 & 0.13 \\
\hline N II $\lambda 5676$ & 25 & 2 & 7.91 & 0.07 \\
\hline N II $\lambda 5679$ & 59 & 3 & 7.86 & 0.06 \\
\hline N II $\lambda 5007$ & 25 & 4 & 7.85 & 0.14 \\
\hline N II $\lambda 5005$ & 50 & 5 & 7.84 & 0.11 \\
\hline \multirow[t]{2}{*}{ N III $\lambda 4634$} & 30 & 7 & 7.81 & 0.25 \\
\hline & $\Delta \zeta_{\mathrm{t}}(\mathrm{\Gamma}$ & & $\begin{array}{c}\epsilon_{\mathrm{N}}=7.86 \\
\Rightarrow\end{array}$ & $\begin{array}{l}\Delta \epsilon_{\mathrm{N}}(\sigma)=0.05 \\
\Delta \epsilon_{\mathrm{N}}\left(\zeta_{\mathrm{t}}\right)=0.02\end{array}$ \\
\hline
\end{tabular}

Notes. Values in bold indicate lines whose abundances deviate by more than $2 \sigma$ from the resulting abundance distribution.

In the three cases, a very low microturbulence $\left(\zeta_{\mathrm{t}}=1 \pm 1 \mathrm{~km} \mathrm{~s}^{-1}\right)$ is required to obtain a zero slope in the $\epsilon_{X}-E W$ diagrams. We have marked in bold, and as gray squares in Fig. 4, the lines whose abundances deviate by more than $2 \sigma$ from the resulting distribution of abundances. These lines

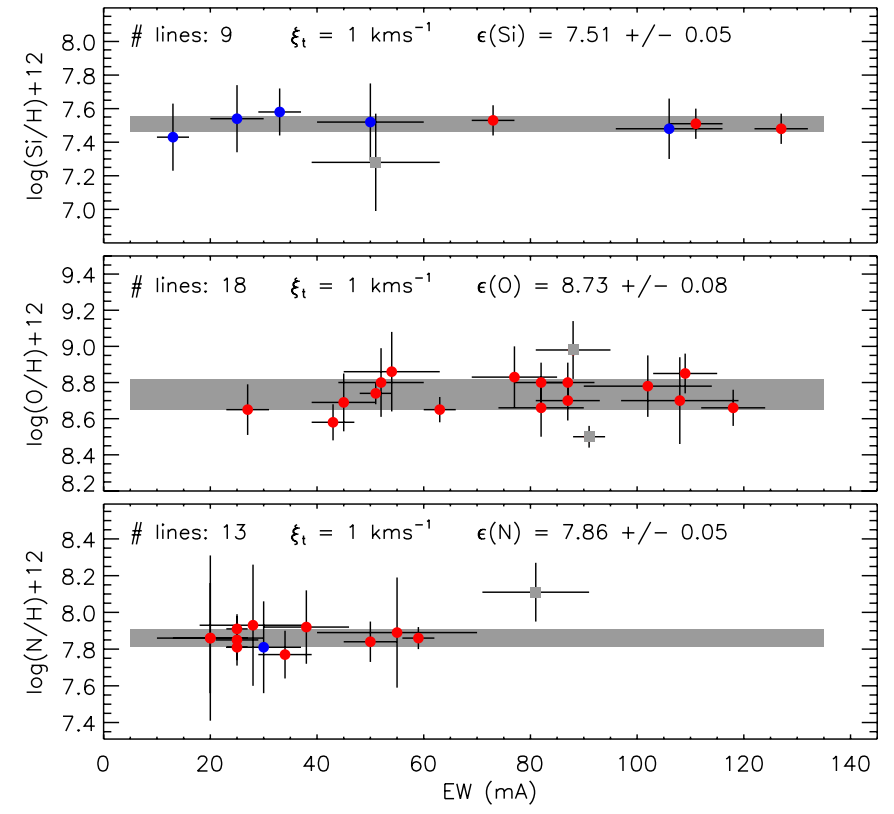

Fig. 4. Final $\log (X / \mathrm{H})$ vs. EW diagrams for $\mathrm{Si}, \mathrm{O}$, and $\mathrm{N}$, resulting from the abundance analysis of $\mathrm{BD}+464374$. The microturbulence providing the lowest dispersion of line-by-line abundances is indicated at the top of each panel along with the associated mean and abundance dispersion. Red and blue dots indicate lines from different ions (red dots: Si III, O II and N II; blue dots: Si IV, and N III); gray dots correspond to the lines excluded from the analysis (see also Sect. 4 and Table 4). The gray horizontal band corresponds to the adopted value and its uncertainty.

were excluded from the final computation of mean abundances $-\epsilon_{\mathrm{X}}-$ and standard deviations $-\Delta \epsilon_{X}(\sigma)-$. We also indicate in Table 4 the uncertainty associated with a change of $\pm 1 \mathrm{~km} \mathrm{~s}^{-1}$ in microturbulence $-\Delta \epsilon_{X}\left(\zeta_{\mathrm{t}}\right)-$ and, for the case of oxygen, the effect of modifying $T_{\text {eff }}$ and $\log g$ by $\pm 1000 \mathrm{~K}$ and $0.1 \mathrm{dex}-$ $\Delta \epsilon_{X}(\mathrm{SP})-$.

\section{Discussion}

\subsection{Nebular and stellar abundances: Cocoon vs. Orion and $M 43$}

The study of interstellar absorption lines of the cold gas by Sofia \& Meyer (2001) demonstrated that the local interstellar medium out to $1.5 \mathrm{Kpc}$ from the Sun is chemically homogeneous to the $10 \%$ level. A similar result has more recently been obtained by Nieva \& Przybilla (2012) from a very thoughtful abundance analysis of a sample of 29 early B-type stars located $500 \mathrm{pc}$ around the Sun. Given its distance to the Sun $(\sim 1 \mathrm{Kpc})$, the Cocoon nebula and BD+46 3474 are expected to share the same chemical composition as the Orion nebula $(d \sim 450 \mathrm{pc})$ and other B-type stars in the solar vicinity. For a discussion about the comparison of chemical abundances from B-type stars in the solar neighborhood and abundances obtained in the Sun, we refer the to the study of Nieva \& Przybilla (2012), who made this comparison in the framework of recent observational data and Galactic chemical and kinematical evolution models.

In the first two columns of Table 5 we present a summary of the Cocoon gas-phase nebular and stellar abundances that were considered below. As discussed in Sect. 3.4, we assumed the weighted average of the gas-phase abundances for apertures 2 
A\&A 571, A93 (2014)

Table 5. Comparison of derived stellar and gas-phase nebular abundances obtained for the Cocoon nebula, M 43, and the Orion nebula.

\begin{tabular}{ccccccc}
\hline \hline & \multicolumn{2}{c}{ Cocoon $^{a}$} & \multicolumn{2}{c}{ Orion } & M 43 & Solar neighborhood \\
\cline { 2 - 6 } & Nebular (CEL) & B stars & Nebular (CEL) $)^{b}$ & B stars & Nebular (CEL) $)^{f}$ & B stars $^{g}$ \\
\hline Si & - & $7.51 \pm 0.05$ & - & $7.51^{c} / 7.50^{d} \pm 0.05$ & - & $7.50 \pm 0.05$ \\
$\mathrm{O}$ & $8.52 \pm 0.05$ & $8.73 \pm 0.08$ & $8.54 \pm 0.03$ & $8.73^{c} / 8.77^{d} \pm 0.04$ & $8.53 \pm 0.09$ & $8.76 \pm 0.05$ \\
$\mathrm{~N}$ & $7.81 \pm 0.03$ & $7.86 \pm 0.05$ & $7.73 \pm 0.09$ & $7.82^{d} \pm 0.07$ & $7.82 \pm 0.04$ & $7.79 \pm 0.04$ \\
$\mathrm{~S}$ & $6.81 \pm 0.04$ & - & $6.83 \pm 0.04$ & $7.15^{e} \pm 0.05$ & $6.95 \pm 0.09$ & - \\
\hline
\end{tabular}

Notes. All the CEL abundances are for $t^{2}=0$. Abundances from other B-type stars in the Solar neighborhood are also quoted for reference. ${ }^{(a)}$ This work ; ${ }^{(b)}$ Reanalysis of the Orion nebula spectrum by Esteban et al. (2004) using the same atomic dataset as in this work (see Table 2); ${ }^{\left({ }^{c}\right)}$ SimónDíaz (2010), similar analysis as in this work using FASTWIND; ${ }^{(d)}$ Nieva \& Simón-Díaz (2011), reanalysis of the spectra from Simón-Díaz (2010) using similar techniques as in Nieva \& Przybilla (2012); ${ }^{(e)}$ Daflon et al. (2009); ${ }^{(f)}$ Reanalysis of M 43 spectrum by Simón-Díaz et al. (2011b) using the same atomic dataset as in this work; ${ }^{(g)}$ Nieva \& Przybilla (2012).

to 4 to be representative of the nebula. For comparative purposes, we also quote:

- the N, O and S abundances resulting from a reanalysis of the Orion nebula and M43 spectra presented in Esteban et al. (2004) and Simón-Díaz et al. (2011a) using the same atomic dataset as in this paper;

- the Si, O, and N abundances derived by Simón-Díaz (2010), Nieva \& Simón-Díaz (2011) for B-type stars in the Orion star-forming region and by Nieva \& Przybilla (2012) for B-type stars in the solar vicinity; and

- the S abundances derived by Daflon et al. (2009) for B-type stars in the Orion star-forming region.

The agreement between the $\mathrm{Si}, \mathrm{O}$, and $\mathrm{N}$ abundances derived from the spectroscopic analysis of BD+46 3474 and the recent determinations of abundances in B-type stars in the Orion OB1 association and, more generally, the solar neighborhood is quite remarkable.

The comparison of gas-phase abundances in the Cocoon nebula and the Orion star-forming region (Orion nebula+M43) is also almost perfect when the same atomic datasets are used (see, however, the effect of assuming different available atomic data for sulphur in Sect. 5.2). Particularly, the comparison with the results obtained for M43 is of special interest, because no ICFs are needed to compute $\mathrm{O}, \mathrm{N}$, and S abundaces in M 43 (see Simón-Díaz et al. 2011b). Using the same atomic dataset as in this work, the abundances of $\mathrm{O}$ and $\mathrm{N}$ are in excellent agreement between both nebulae (see Table 5). The differences between $\mathrm{S}$ abundances in the Cocoon nebula and in M 43 cannot be attributed to atomic data or to the use of an ICF; however, we used different lines to compute $\mathrm{S}^{2+} / \mathrm{H}^{+}$ratio: in the Cocoon nebula we used the bright nebular lines at $\lambda \lambda 9069,9531$, while in M $43^{8}$ we used the faint and extremely temperature-dependent auroral line at $\lambda 6312$. Because we assumed the same temperature for the $\mathrm{S}^{+}$and $\mathrm{S}^{2+}$ regions $\left(T_{\mathrm{e}}\left(\left[\mathrm{N}_{\mathrm{II}}\right]\right)\right.$ for the Cocoon nebula and $T_{\mathrm{e}}([\mathrm{OII}])$ for $\left.\mathrm{M} 43\right)$, differences in the true temperature in the zones where the different ions are present may explain the observed discrepancy. This shows the importance of using consistent sets of lines, physical conditions, atomic data, and ICFs when comparing abundances obtained for the same element in different objects.

For the Orion nebula, there is a small difference between $\mathrm{N}$ abundances, which may be perfectly explained by uncertainty

\footnotetext{
8 The spectra of M43 used by Simón-Díaz et al. (2011b) do not cover the near-infrared zone of the spectrum where the bright nebular [SIII] lines lie.
}

in the $\operatorname{ICF}(\mathrm{N})$ that has to be assumed for the Orion nebula. We only indicate in Table 5 the value provided by García-Rojas \& Esteban (2007) but Esteban et al. (2004) and Simón-Díaz \& Stasińska (2011) proposed another two possible values of the total gas-phase $\mathrm{N}$ abundance in the Orion nebula $(7.65 \pm 0.09$ and $7.92 \pm 0.09)$. The three values result from the analysis of the same spectrum but assuming a different $\operatorname{ICF}(\mathrm{N})$. In this context, we highlight the importance of the Cocoon nebula and M43 for comparing nitrogen abundances derived from the analysis of the nebular and stellar spectra (Sect. 5.2), since in these cases no $\operatorname{ICF}(\mathrm{N})$ is needed to obtain the total nebular nitrogen abundance. The results obtained for these two nebulae strongly favor the ICF(N) used by García-Rojas \& Esteban (2007) for computing total $\mathrm{N}$ abundance in the Orion nebula.

\subsection{Nebular vs. stellar abundances in the Cocoon nebula}

Before comparing nebular and stellar abundances in the Cocoon nebula, we briefly summarize the main results of a similar study performed by Simón-Díaz \& Stasińska (2011) in the Orion starforming region. They used the chemical abundance study of the Orion star-forming region from B-type stars (Simón-Díaz 2010; Nieva \& Simón-Díaz 2011) to compare the derived abundances with those obtained for non-refractory elements $(\mathrm{C}, \mathrm{N}, \mathrm{O}$, and $\mathrm{Ne}$ ) in the most detailed study of the gas-phase chemical abundances on the Orion nebula (Esteban et al. 2004). The main conclusion of these authors is that oxygen abundance derived from CELs (corrected for depletion onto dust grains) in the Orion nebula is irreconcilable with that derived from B-type stars. On the other hand, they found that $\mathrm{N}$ and $\mathrm{Ne}$ gas phase abundances and $\mathrm{C}$ gas phase+dust abundances from CELs seemed to be consistent with those derived in B-type stars (see Fig. 1 of Simón-Díaz \& Stasińska 2011). In addition, these authors found that oxygen gas phase+dust abundances derived from optical recombination lines (ORLs) agree very well with oxygen abundances derived in the stars.

In the study of the Cocoon nebula presented here we concentrate on the comparison of nebular and stellar abundances for $\mathrm{O}, \mathrm{N}$, and S. As indicated in Sects. 3.4 and 5.1, one important point of this study (compared with the case of the Orion nebula) is that the total abundances of the three investigated elements are obtained without the necessity of any ICF. Although we only have access to nebular abundances derived from CELs (no ORLs are detected), we also include in our discussion how CEL abundances corrected from the presence of possible temperature fluctuations compare with the stellar abundances. In particular, since 
we cannot directly compute the $t^{2}$ parameter from our observations, we assume two cases: a canonical value of $t^{2}=0.035$ (which is an average value in Galactic H II regions, see GarcíaRojas \& Esteban 2007), and the value derived for the Orion nebula $\left(t^{2}=0.022\right.$, Esteban et al. 2004).

A meaningful comparison of nebular and stellar abundances first requires the nebular gas-phase abundances (Tables 1 and 5) to be corrected for possible depletion onto dust grains. Several authors have estimated the oxygen deplection factor in the Orion nebula by comparing abundances of the refractory elements $\mathrm{Mg}$, $\mathrm{Si}$, and $\mathrm{Fe}$ in the gas phase with those found in the atmospheres of B stars of the Orion cluster (e.g. Esteban et al. 1998; MesaDelgado et al. 2009a; Simón-Díaz \& Stasińska 2011); these authors found oxygen depletions between 0.08 and 0.12 dex. For a detailed discussion on the computations of such depletions, we refer to Simón-Díaz \& Stasińska (2011). Because we lacked the information needed to perform a similar computation in the Cocoon nebula, we decided to adopt a canonical value of $0.10 \mathrm{dex}$ as representative of the oxygen depletion in this nebula and considered an associated uncertainty of \pm 0.02 dex. Nitrogen is expected to be a minor constituent of dust in H II regions (Jenkins 2014); therefore, no correction is needed. For sulphur, the situation is more complicated; although for a long time sulphur was thought not to be depleted onto dust grains (see, e.g. Sofia et al. 1994), recently, some authors (Jenkins 2009; White $\&$ Sofia 2011) drew attention to the risks of assuming sulphur as a standard for what should be virtually zero depletion, especially for some sight lines. Unfortunately, there is a lack of quantitative studies on the sulphur depletion onto dust grains, which makes this an open question that needs to be addressed in the future by using high-quality interstellar abundance measurements. We hence assumed no dust correction for sulphur, but kept in mind this argument.

\subsubsection{Oxygen}

Similarly to the case of the Orion nebula, the derived gas+dust oxygen abundance resulting from CELs and a $t^{2}=0$ $(8.62 \pm 0.05)$ is remarkably different from the $\mathrm{O}$ abundance obtained from the spectroscopic analysis of the central star. If we consider as valid the assumption that temperature fluctuations affect the determination of ionic chemical abundances using CELs (Peimbert 1967; Peimbert \& Costero 1969), and the canonical value of $t^{2}=0.035$, we would obtain that the total gas+dust nebular abundance would reach $12+\log (\mathrm{O} / \mathrm{H})=8.86$, which is now much higher than the abundance obtained from stars. While this result could be used as an argument against the temperature fluctuation scheme, we recall that we considered a value of $t^{2}$ that may not be representative of the actual value in the Cocoon nebula. In particular, if the value of $t^{2}$ derived for the Orion nebula is considered (0.022), the resulting gas+dust oxygen abundance would be $12+\log (\mathrm{O} / \mathrm{H})=8.75$, in much better agreement with the stellar abundance.

\subsubsection{Nitrogen}

As Simón-Díaz \& Stasińska (2011) argued, if the RL-CEL abundance discrepancy were caused by temperature fluctuations, as suggested by Peimbert et al. (1993), one should observe the same kind of bias in the CEL abundances of the other elements. They did not find other elements such as N, C, and Ne following the same behavior as oxygen but they also claimed that the derived total gas-phase abundances of $\mathrm{C}, \mathrm{N}$, and $\mathrm{Ne}$ in the Orion nebula are much less accurate. This is mainly due to the uncertainties on the adopted ICFs. In the Cocoon nebula, no ICF correction needs to be applied to compute the total $\mathrm{N}$ gas-phase abundance. This is due to the low excitation of the nebula, which prevents the ionization of $\mathrm{N}^{+}$to $\mathrm{N}^{2+}$. From the comparison of the total nebular $\mathrm{N}$ abundance obtained from CELs (and $t^{2}=0$ ), $12+\log (\mathrm{N} / \mathrm{H})=7.81 \pm 0.03$ and that obtained from the analysis of the central star, $12+\log (\mathrm{N} / \mathrm{H})=7.86 \pm 0.05$, we can conclude that the stellar abundance is slightly higher, but both values are consistent within the uncertainties. In this case, the CEL $+t^{2}$ abundances that result from assuming a $t^{2}=0.035$ (canonical) or 0.022 (Orion nebula) are 8.02 and $7.93^{9}$. The latter option also agrees with the stellar solution.

For completeness, in this section we caution regarding the stellar nitrogen abundance. Spectroscopic analyses of early-B type main-sequence stars in the past years have shown increasing observational evidence of the existence of a significant percentage of narrow-lined (low $v \sin i$, but not neccesarily fast rotators seen pole-on) targets among these stars showing nitrogen enhancement in their photospheres (e.g., Morel et al. 2006; Hunter et al. 2008). This result indicates the danger of extracting any conclusion from the direct comparison of nebular and stellar abundances based on one target. We hence must consider the derived nitrogen abundance in BD+46 3474 as an upper limit to be compared with the nebular abundance, especially in view of the nitrogen abundance obtained for this star in comparison with other stars in the solar neighborhood (see Table 5).

\subsubsection{Sulphur}

Given the low excitation of the Cocoon nebula, which prevents the presence of ionization species of $\mathrm{S}$ higher than $\mathrm{S}^{2+}$, we skipped the uncertainty associated with the use of an ICF to compute the total nebular sulphur abundance ${ }^{10}$. However, there are still some problems that make the comparison of nebular and stellar abundances for this element uncertain. First, there is the remarkable difference in the computed abundances when assuming different atomic datasets. To illustrate this, we recomputed the Coocon nebular sulphur abundance using the same atomic data for this element as García-Rojas \& Esteban (2007) ${ }^{11}$. The value proposed by these authors for the sulphur abundance in the Orion nebula was $7.04 \pm 0.04$, a factor 1.6 higher than the abundance indicated in Table 5. For the Cocoon nebula, the abundance is modified from 6.81 to 6.90 (i.e., $20 \%$ difference). Second, as discussed above, there are doubts about the depletion of sulphur on dust (Jenkins 2009), and some amount of it may be in the form of dust grains. In addition, we still do not have implemented and tested a sulphur model atom to be used with FASTWIND and could not derive the $\mathrm{S}$ abundance associated with $\mathrm{BD}+463474$; however, given the good match between the $\mathrm{Si}, \mathrm{O}$, and $\mathrm{N}$ abundances in $\mathrm{BD}+463474$ and other B-type stars in the Orion star-forming region (Sect. 5.1), we consider

9 Note that the correction to the abundances derived from [N II] CELs is much lower than that derived from [O II] CELs. In particular, this correction depends on the wavelength of the used lines for abundance calculations, which is higher for bluer lines, such as [O II] $\lambda \lambda 3726+29$ and lower for redder lines, such as [N II] $\lambda \lambda 6548+83$.

${ }^{10}$ Taking into account the lack of $\mathrm{O}^{2+}$ in the nebula and the similarity between ionization potentials of $\mathrm{O}^{+}(35.12 \mathrm{eV})$ and $\mathrm{S}^{2+}(34.83 \mathrm{eV})$, this seems to be a reasonable conclusion.

11 The atomic dataset used by these authors was the following: collision strengths by Ramsbottom et al. (1996) for $\mathrm{S}^{+}$and Tayal \& Gupta (1999) for $\mathrm{S}^{2+}$. Transition probabilities by Keenan et al. (1993) for $\mathrm{S}^{+}$ and Mendoza \& Zeippen (1982) and Kaufman \& Sugar (1986) for $\mathrm{S}^{2+}$. 
Table 6. Summary of the abundances presented in Fig. 5.

\begin{tabular}{|c|c|c|c|c|c|c|c|c|}
\hline \multicolumn{5}{|c|}{ Cocoon } & \multicolumn{4}{|c|}{ Orion } \\
\hline & \multicolumn{3}{|c|}{ Nebular gas+dust (CEL) } & \multirow[t]{2}{*}{ B stars } & \multicolumn{2}{|c|}{ Nebular gas+dust (CEL) } & \multirow{2}{*}{$\begin{array}{c}\text { Nebular } \\
\text { gas+dust (ORL) }\end{array}$} & \multirow[t]{2}{*}{ B stars } \\
\hline & $t^{2}=0.00$ & $t^{2}=0.022$ & $t^{2}=0.035$ & & $t^{2}=0.00$ & $t^{2}=0.022$ & & \\
\hline$\overline{\mathrm{O}}$ & $8.62 \pm 0.05$ & $8.75 \pm 0.05$ & $8.86 \pm 0.05$ & $8.73 \pm 0.08$ & $8.64 \pm 0.03$ & $8.75 \pm 0.03$ & $8.73 \pm 0.03$ & $8.75 \pm 0.05$ \\
\hline $\mathrm{N}$ & $7.81 \pm 0.03$ & $7.93 \pm 0.03$ & $8.02 \pm 0.03$ & $7.86 \pm 0.05$ & $7.73 / 7.92 \pm 0.03^{1}$ & $7.81 / 8.00 \pm 0.03^{1}$ & - & $7.82 \pm 0.07$ \\
\hline$S$ & $6.81 / 6.88 \pm 0.04^{2}$ & $6.96 / 7.03 \pm 0.04^{2}$ & $7.07 / 7.14 \pm 0.04^{2}$ & - & $6.83 / 7.04 \pm 0.04^{2}$ & $6.99 / 7.20 \pm 0.04^{2}$ & - & $7.15 \pm 0.05$ \\
\hline
\end{tabular}

Notes. ${ }^{(1)}$ Two ICFs used (see text). ${ }^{(2)}$ Two sets of atomic data used (see text).

the comparison of our nebular sulphur abundance with those obtained by Daflon et al. (2009) as still a valid exercise ${ }^{12}$.

From the comparison of the sulphur abundances presented in Table 5 we conclude that there is a clear discrepancy (by more than 0.3 dex) between the nebular CEL $\left(t^{2}=0\right)$ and stellar abundances. If we consider the temperature fluctuations and assume the canonical value for Galactic H II regions and that of the Orion nebula, we would overcome a large part of the discrepancy, reaching values of $12+\log (\mathrm{S} / \mathrm{H})=7.07$ and 6.96 (to be compared with $7.15 \pm 0.05$ ). While these values agree much better with the stellar abundance, the $t^{2}=0.022$ solution (the one for which we find better agreement for of $\mathrm{O}$ and $\mathrm{N}$ ) is still far away from the value resulting from the analysis of the stellar spectra.

Interestingly, a perfect agreement between nebular and stellar sulphur abundances would be obtained if the atomic dataset considered by García-Rojas \& Esteban (2007) were assumed and combined with a $t^{2}=0.035$. However, while still a valid option, this possibility is highly speculative and far from being considered as a valid scientific argument supporting any conclusion.

\subsubsection{Final remarks}

In Fig. 5 we illustrate this discussion by showing the comparison between the abundances of $\mathrm{O}, \mathrm{N}$, and $\mathrm{S}$ in the Cocoon nebula obtained using CELs and $t^{2}=0$, CELs and $t^{2}=0.022$ (the Orion nebula value) and CELs and $t^{2}=0.035$ (canonical $t^{2}$ value for Galactic H II regions) with the abundances in the central star $\mathrm{BD}+463474$. For comparison we also represent the values for CELs and $t^{2}=0$ and $t^{2}=0.022$ and the oxygen ORLs values for the Orion nebula. All these numbers are summarized in Table 6. As a general result, for $\mathrm{O}$ and $\mathrm{N}$, it is clear that abundances considering small temperature fluctuations $\left(t^{2}=0.022\right.$, similar to what is found in the Orion nebula) agree much better with what is obtained from stars than the abundances considering pure CELs with $t^{2}=0$. On the other hand, typical values of $t^{2}=0.035$ found in Galactic H II regions seem to overestimate nebular $\mathrm{O}$ and $\mathrm{N}$ abundance. As representative of the ICF problem, in Fig. 5 we show two values of the Orion $\mathrm{N}$ abundance, assuming the ICF by García-Rojas \& Esteban (2007) and Simón-Díaz \& Stasińska (2011); it is evident that by using different ICFs one can reach radically different conclusions.

For sulphur, the situation is puzzling. Although some increase of the $\mathrm{S}$ abundance owing to depletion onto dust cannot be ruled out, it seems that the results for CELs and $t^{2}=0$ are

\footnotetext{
${ }^{12}$ More recently, Irrgang et al. (2014) included three of the Orion stars analyzed in Simón-Díaz (2010) using a similar technique and atomic data as Nieva \& Simón-Díaz (2011). The resulting sulphur abundances agree well with the values obtained by Daflon et al. (2009).
}

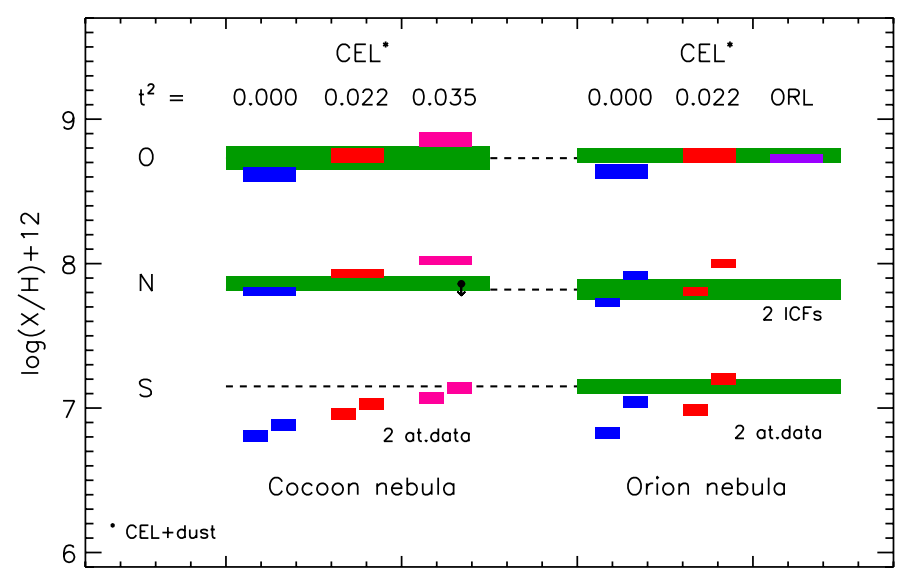

Fig. 5. Comparison of $\mathrm{O}, \mathrm{N}$, and $\mathrm{S}$ abundances obtained from B-type stars (green) and nebular (gas+dust) abundances derived from CEL and different values of $t^{2}$ (blue, red, and magenta). The violet box indicates $\mathrm{O}$ abundance obtained from ORLs in the Orion nebula by Esteban et al. (2004). The height of the boxes represents the uncertainties. Left and right columns correspond to the Cocoon and the Orion nebula. For sulphur, the resulting abundances using two different atomic datasets are indicated; for nitrogen in the Orion nebula, the abundances computed by assuming two different ICFs are shown. See text for more details.

far from stellar abundances. Additionally, the abundances are highly dependent on the selected atomic dataset (especially for the Orion nebula, where $\mathrm{S}^{2+}$ is dominant). We computed sulphur nebular abundances using atomic data from Table 2 (left) and atomic data used in García-Rojas \& Esteban (2007). In general, by using the atomic dataset of Table 2, sulphur nebular abundances are far from the stellar results assuming $t^{2}=0.022$ in both, the Cocoon and the Orion nebula, but can agree assuming $t^{2}=0.035$, which was considered to be too high from the analysis of $\mathrm{O}$ and $\mathrm{N}$ data. On the other hand, assuming the atomic dataset of García-Rojas \& Esteban (2007), the situation changes and one can reconcile sulphur nebular and stellar abundances by assuming $t^{2}=0.022$. Of course, this result does not necessarily favor one given dataset, but warns about the influence of atomic data in our results (see Luridiana et al. 2011; Luridiana \& García-Rojas 2012, for a critical review on the use of nebular atomic data).

All these results do not necessarily imply the existence of such temperature fluctuations, regardless of the physical origin of such fluctuations, but they warn us about the use of pure CELs as a proxy for computing chemical abundances in photoionized regions. Additionally, special care should be taken when selecting an ICF scheme and/or an atomic dataset, owing to a poor 
choice, could reach large uncertainties on the nebular chemical abundances and hence, to incorrect interpretations.

We emphasize the importance of including more elements to compare in future studies (mainly $\mathrm{C}, \mathrm{Ne}$, and $\mathrm{Ar}$ ). The main problem with these elements is that we would always need an ICF to compute the total nebular abundance from optical spectra. This problem may be circumvented using multiwavelength studies including UV and IR lines, but this is very difficult for very extended objects, such as Galactic H II regions owing to the different apertures used for UV, optical, and IR spectrographs, which may introduce considerable ionization structure effects. A detailed set of photoionization models covering as much as possible of the H II regions parameter space is needed to build a consistent set of ICFs, as has recently been done for planetary nebulae by Delgado-Inglada et al. (2014).

\section{Summary and conclusions}

The Cocoon nebula (IC 5146) - a roundish H II region ionized by a single B0.5 V star (BD+463474) - seems to be an ideal object to compare stellar and nebular chemical abundances and then check the abundance determinations methods in the field of $\mathrm{H}$ II regions and massive stars.

We collected a set of high-quality observations comprising the optical spectrum of $\mathrm{BD}+463474$ (the main ionizing source), along with long-slit spatially resolved nebular spectroscopy of the nebula.

We presented the nebular abundance analysis of the spectra extracted from apertures located at various distances from the central star in the Cocoon nebula, and a quantitative spectroscopical analysis of the ionizing central star BD+463474.

We performed a detailed nebular empirical analysis of eight apertures extracted from a long-slit located to the northwest of $\mathrm{BD}+463474$. We obtained the spatial distribution of the physical conditions (temperature and density) and ionic abundances of $\mathrm{O}^{+}, \mathrm{N}^{+}, \mathrm{S}^{+}$and $\mathrm{S}^{2+}$. Owing to the extremely low ionization degree of the Cocoon nebula, we can determine total abundances directly from observable ions, eliminating the uncertainties resulting of assuming an ICF scheme, which are especially significant for $\mathrm{N}$. In particular, the $\mathrm{N}$ abundance completely agrees with that determined by Simón-Díaz et al. (2011b) for M43, a local $\mathrm{H}$ II region with a similar low ionization degree.

By means of a quantitative spectroscopic analysis of the optical spectrum of BD+463474 with the stellar atmosphere code FASTWIND, we derived for this B0.5 V star $T_{\text {eff }}=30500 \pm 1000 \mathrm{~K}$ and $\log g=4.2 \pm 0.1$. and chemical abundances of $\mathrm{Si}, \mathrm{O}$, and $\mathrm{N}$ (in $12+\log (X / \mathrm{H})$ ) of $7.51 \pm 0.05$, $8.73 \pm 0.08$, and $7.86 \pm 0.05$, respectively.

From the comparison of the $\mathrm{O}, \mathrm{N}$, and $\mathrm{S}$ abundances in the nebula and in its central star we conclude that i) abundances derived from CELs are, in general, lower than those found in stars for the same element; ii) considering moderate temperature fluctuations, similar to what is found in the Orion nebula $\left(t^{2}=0.022\right)$, and dust depletion for $\mathrm{O}$, we would reconcile the abundances in the nebula and the central star for O and N. For $\mathrm{S}$, the results are somewhat puzzling and point to different conclusions depending on the atomic dataset adopted for computing the ionic abundances.

As a future step, this type of study should be extended to other elements and $\mathrm{HII}$ regions with the aim of looking for systematic effects in the nebular and stellar abundances. Multiwavelength nebular studies taking into account aperture effects and/or a new set of theoretical ICFs from a complete grid of H II region photoionization models as well as multielement abundance studies from a large number of massive stars in the same star-forming region would minimize uncertainties and probably would shed some light on this still poorly explored topic.

Acknowledgements. This work has been funded by the Spanish Ministry of Economy and Competitiveness (MINECO) under the grants AYA2010-21697C05-04, AYA2011-22614, and AYA2012-39364-C02-01, and by the Canary Islands Government under grant PID2010119. JGR and SSD acknowledge support from Severo Ochoa excellence program (SEV-2011-0187) postdoctoral fellowships. We thank A. R. López-Sánchez for kindly providing us the image for Fig. 1. We also thank the anonymous referee for the suggestions.

\section{Appendix A: Distance to the Cocoon nebula as determined from $B D+463474$}

There have been several independent determinations of the distance to the IC 5146 star-forming region, where the Cocoon nebula is located. The proposed values ranges from 460 to $1400 \mathrm{pc}$. We refer to Harvey et al. (2008) for a complete compilation of published distance estimates previous to 2008, and a detailed discussion of the various considered methodologies and their reliability.

In this paper, we were mainly interested in the quantitative spectroscopic analysis of BD+46 3474 to determine its photospheric chemical composition and compare the derived abundances with those resulting from the study of the Cocoon nebula spectrum. However, as an additional result, we also reevaluate the question of the distance to this star and its associated H II region using state-of-the-art information. Below, we describe the methodology we have followed and our proposed value.

Table 3 summarized the spectroscopic parameters $\left(T_{\text {eff }}\right.$ and $\log g$, among others) resulting from the FASTWIND analysis, as well as some photometric information that we used to evaluate the distance (the $V$ magnitude and the $B-V$ color). From the comparison of intrinsic $(B-V)_{0}$ color predicted by a FASTWIND model with the indicated $T_{\text {eff }}$ and $\log g$ and the observed value, we obtained the value of the extinction parameter in the $V$ band. We hence determined $M_{v}, R, \log L$, and $M_{\mathrm{sp}}$ (spectroscopic mass) assuming several distance values. The absolute visual magnitude was computed by means of

$M_{v}=V-5 \log d+5-A_{v}$

and the stellar radius, luminosity, and spectroscopic mass was derived by means of the strategy indicated in Herrero et al. (1992). Last, we located the star in the HR diagram and computed the evolutionary tracks by Brott et al. (2011).

Table A.1 and Fig. A.1 summarizes the results from this exercise. From inspection of Fig. A.1 it becomes clear that any distance below $720 \mathrm{pc}$ is not possible since the star would be located below the zero-age main sequence (ZAMS) line. We selected four distances above that value. The largest one $(1.2 \mathrm{Kpc})$ is the value proposed by Herbig \& Dahm (2002) based on the spectroscopic distances to the late-B stars and two different main-sequence calibrations: the Jaschek \& Gomez (1998) absolute magnitudes for B dwarf standards, and the Schmidt-Kaler ZAMS (Aller et al. 1982). The other values are our suggested distance $(800 \mathrm{pc})$, the value proposed by Harvey et al. (2008), and an intermediate value.

As illustrated in Table A.1 and Fig. A.1, the determined spectroscopic mass, evolutionary mass, and age are a function of the assumed distance. We can clearly discard the $1200 \mathrm{pc}$ (and even the $950 \mathrm{pc}$ ) solutions since these distances result in a very poor 
Table A.1. Computed values of absolute visual magnitude, stellar radius, luminosity, spectroscopic mass, evolutionary mass, and age for different assumed distances to BD+46 3474 (see Sect. A for explanations).

\begin{tabular}{c|cccccc}
\hline \hline$d(\mathrm{pc})$ & $M_{\mathrm{v}}$ & $R\left(R_{\odot}\right)$ & $\log \left(L / L_{\odot}\right)$ & $M_{\mathrm{sp}}\left(M_{\odot}\right)$ & $M_{\mathrm{ev}}\left(M_{\odot}\right)$ & Age $(\mathrm{Myr})$ \\
\hline 720 & -2.8 & 4.7 & 4.23 & 13 & $14-15$ & ZAMS \\
$\mathbf{8 0 0}^{1}$ & $-\mathbf{3 . 0}$ & $\mathbf{5 . 2}$ & $\mathbf{4 . 3 1}$ & $\mathbf{1 5}$ & $\sim \mathbf{1 5}$ & $\mathbf{2}$ \\
850 & -3.2 & 5.5 & 4.37 & 18 & $15-16$ & 3 \\
950 & -3.4 & 6.2 & 4.47 & 22 & $\sim 16$ & 4 \\
1200 & -3.9 & 7.8 & 4.68 & 35 & $\sim 18$ & 6 \\
\hline
\end{tabular}

Notes. ${ }^{(1)}$ We indicate in bold our proposed distance.

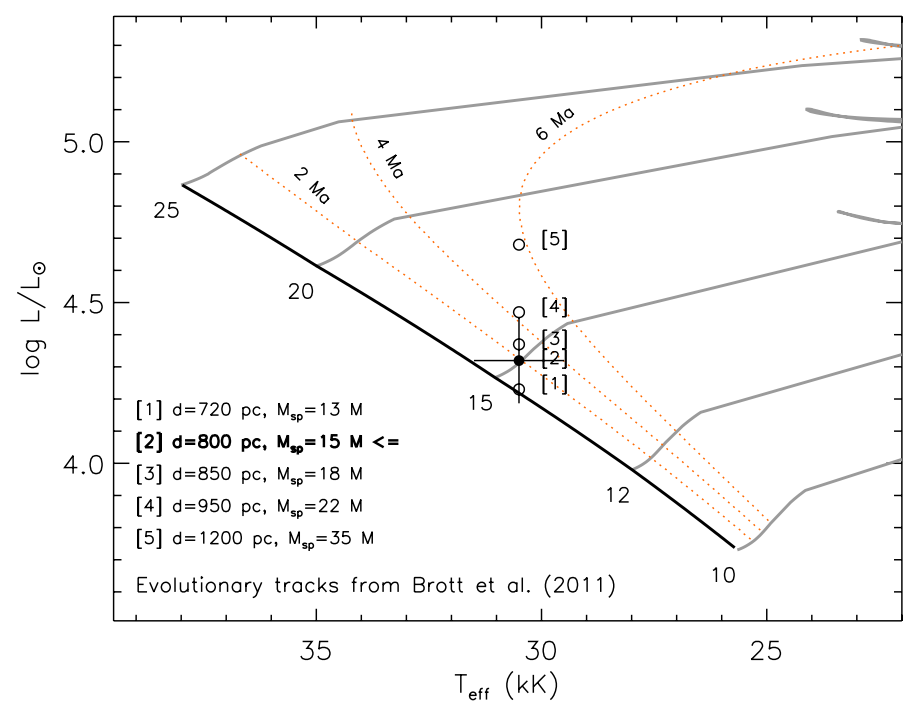

Fig. A.1. Location of BD+46 3474 in the HR diagram for different values of assumed distance (see also Table A.1). Evolutionary tracks and isocrones from Brott et al. (2011). The corresponding spectroscopic masses are also indicated.

agreement between the spectroscopic and evolutionary masses and a too evolved star ( $\geq 4 \mathrm{Myr}$ ). Note that given the high number of accreting pre-MS stars, we expect the IC 5146 cluster to be younger than a few Myr (Herbig \& Dahm 2002; Harvey et al. 2008). We hence use the $M_{\mathrm{sp}}=M_{\mathrm{ev}}$ criterium to propose $800 \pm 80 \mathrm{pc}$ as the distance to BD+46 3474 .

\section{References}

Aller, L. H., Appenzeller, I., Baschek, B., et al. 1982, Landolt-Bornstein: Group 6: Astronomy (Berlin, Heidelberg, New york: Springer - Verlag) Bresolin, F., Gieren, W., Kudritzki, R.-P., et al. 2009, ApJ, 700, 309 Brott, I., de Mink, S. E., Cantiello, M., et al. 2011, A\&A, 530, A115 Carigi, L., Peimbert, M., Esteban, C., \& García-Rojas, J. 2005, ApJ, 623, 213 Chiappini, C., Romano, D., \& Matteucci, F. 2003, MNRAS, 339, 63

Daflon, S., Cunha, K., de la Reza, R., Holtzman, J., \& Chiappini, C. 2009, AJ, 138,1577

Delgado-Inglada, G., Morisset, C., \& Stasińska, G. 2014, MNRAS, 440, 536 Esteban, C., Peimbert, M., Torres-Peimbert, S., \& Escalante, V. 1998, MNRAS, 295, 401

Esteban, C., Peimbert, M., Torres-Peimbert, S., \& Rodríguez, M. 2002, ApJ, 581, 241

Esteban, C., Peimbert, M., García-Rojas, J., et al. 2004, MNRAS, 355, 229

Esteban, C., García-Rojas, J., Peimbert, M., et al. 2005, ApJ, 618, L95

Esteban, C., Bresolin, F., Peimbert, M., et al. 2009, ApJ, 700, 654

Esteban, C., Carigi, L., Copetti, M. V. F., et al. 2013, MNRAS, 433, 382

Esteban, C., García-Rojas, J. Carigi, L., et al. 2014, MNRAS, 443, 624
Ferland, G. J. 1999, PASP, 111, 1524

Gail, H.-P., \& Sedlmayr, E. 1986, A\&A, 166, 225

Galavís, M. E., Mendoza, C., \& Zeippen, C. J. 1995, A\&AS, 111, 347 Galavis, M. E., Mendoza, C., \& Zeippen, C. J. 1997, A\&AS, 123, 159 García-Rojas, J., \& Esteban, C. 2007, ApJ, 670, 457

Harvey, P. M., Huard, T. L., Jørgensen, J. K., et al. 2008, ApJ, 680, 495 Henry, R. B. C., Edmunds, M. G., \& Köppen, J. 2000, ApJ, 541, 660 Herbig, G. H., \& Dahm, S. E. 2002, AJ, 123, 304

Herrero, A., Kudritzki, R. P., Vilchez, J. M., et al. 1992, A\&A, 261, 209

Hunter, I., Brott, I., Lennon, D. J., et al. 2008, ApJ, 676, L29

Irrgang, A., Przybilla, N., Heber, U., et al. 2014, A\&A, 565, A63

Jaschek, C., \& Gomez, A. E. 1998, A\&A, 330, 619

Jenkins, E. B. 2009, ApJ, 700, 1299

Jenkins, E. B. 2014, in Life Cycle of Dust in the Universe, Observations, Theory and Laboratory Experiments, submitted [arXiv: 1402 .4765]

Kaufman, V., \& Sugar, J. 1986, J. Phys. Chem. Ref., Data, 15, 321

Keenan, F. P., Hibbert, A., Ojha, P. C., \& Conlonl, E. . 1993, Phys. Scr., 48, 129 López-Sánchez, A. R., Esteban, C., García-Rojas, J., Peimbert, M., \& Rodríguez, M. 2007, ApJ, 656, 168

López-Sánchez, A. R., Dopita, M. A., Kewley, L. J., et al. 2012, MNRAS, 426, 2630

Luridiana, V., \& García-Rojas, J. 2012, in IAU Symp. 283, eds. A. Manchado, L. Stanghellini, \& D. Schönberner, 139

Luridiana, V., Simón-Díaz, S., Cerviño, M., et al. 2009, ApJ, 691, 1712

Luridiana, V., García-Rojas, J., Aggarwal, K., et al. 2011 [arXiv: 1110. 1873]

Luridiana, V., Morisset, C., \& Shaw, R. A. 2014, A\&A, in press DOI: 10.1051/0004-6361/201323152

Mendoza, C., \& Zeippen, C. J. 1982, MNRAS, 199, 1025

Mesa-Delgado, A., Esteban, C., García-Rojas, J., et al. 2009a, MNRAS, 395, 855

Mesa-Delgado, A., López-Martín, L., Esteban, C., García-Rojas, J., \& Luridiana, V. 2009b, MNRAS, 394, 693

Mesa-Delgado, A., Núñez-Díaz, M., Esteban, C., et al. 2012, MNRAS, 426, 614 Morel, T., Butler, K., Aerts, C., Neiner, C., \& Briquet, M. 2006, A\&A, 457, 651 Nicholls, D. C., Dopita, M. A., \& Sutherland, R. S. 2012, ApJ, 752, 148

Nicholls, D. C., Dopita, M. A., Sutherland, R. S., Kewley, L. J., \& Palay, E. 2013, ApJS, 207, 21

Nieva, M.-F., \& Przybilla, N. 2012, A\&A, 539, A143

Nieva, M.-F., \& Simón-Díaz, S. 2011, A\&A, 532, A2

Peña-Guerrero, M. A., Peimbert, A., \& Peimbert, M. 2012, ApJ, 756, L14

Peimbert, A. 2003, ApJ, 584, 735

Peimbert, M. 1967, ApJ, 150, 825

Peimbert, M. 2008, Current Science, 95, 1165

Peimbert, M., \& Costero, R. 1969, Boletin de los Observatorios de Tonantzintla y Tacubaya, 5, 3

Peimbert, A., \& Peimbert, M. 2013, ApJ, 778, 89

Peimbert, M., Storey, P. J., \& Torres-Peimbert, S. 1993, ApJ, 414, 626

Peimbert, M., Peimbert, A., Esteban, C., et al. 2007, in Rev. Mex. Astron. Astrofis., 29, 72

Podobedova, L. I., Kelleher, D. E., \& Wiese, W. L. 2009, J. Phys. Chem. Ref. Data, 38, 171

Pradhan, A. K., Montenegro, M., Nahar, S. N., \& Eissner, W. 2006, MNRAS, 366, L6

Puls, J., Kudritzki, R.-P., Herrero, R., et al. 1996, A\&A, 305, 171

Puls, J., Urbaneja, M. A., Venero, R., et al. 2005, A\&A, 435, 669

Ramsbottom, C. A., Bell, K. L., \& Stafford, R. P. 1996, At. Data Nucl. Data Tables, 63, 57

Rivero González, J. G., Puls, J., \& Najarro, F. 2011, A\&A, 536, A58

Rivero González, J. G., Puls, J., Najarro, F., \& Brott, I. 2012, A\&A, 537, A79

Santolaya-Rey, A. E., Puls, J., \& Herrero, A. 1997, A\&A, 323, 488

Simón-Díaz, S. 2010, A\&A, 510, A22 
J. García-Rojas et al.: The Cocoon nebula and its ionizing star

Simón-Díaz, S., \& Herrero, A. 2014, A\&A, 562, A135

Simón-Díaz, S., \& Stasińska, G. 2011, A\&A, 526, A48

Simón-Díaz, S., Castro, N., Herrero, A., et al. 2011a, J. Phys. Conf. Ser., 328, 2021

Simón-Díaz, S., García-Rojas, J., Esteban, C., et al. 2011b, A\&A, 530, A57

Sofia, U. J., \& Meyer, D. M. 2001, ApJ, 554, L221

Sofia, U. J., Cardelli, J. A., \& Savage, B. D. 1994, ApJ, 430, 650

Stasińska, G., Tenorio-Tagle, G., Rodríguez, M., \& Henney, W. J. 2007, A\&A, 471,193

Storey, P. J., \& Hummer, D. G. 1995, MNRAS, 272, 41

Tayal, S. S. 2011, ApJS, 195, 12

Tayal, S. S., \& Gupta, G. P. 1999, ApJ, 526, 544
Telting, J. H., Avila, G., Buchhave, L., et al. 2014, Astron. Nachr., 335, 41

Torres-Peimbert, S., \& Peimbert, M. 1977, Rev. Mex. Astron. Astrofis., 2, 181

Tremonti, C. A., Heckman, T. M., Kauffmann, G., et al. 2004, ApJ, 613, 898

Trundle, C., Dufton, P. L., Lennon, D. J., Smartt, S. J., \& Urbaneja, M. A. 2002, A\&A, 395, 519

Tsamis, Y. G., \& Péquignot, D. 2005, MNRAS, 364, 687

Tsamis, Y. G., Walsh, J. R., Vílchez, J. M., \& Péquignot, D. 2011, MNRAS, 412, 1367

U, Vivian, Urbaneja, M. A., Kudritzki, R.-P., et al. 2009, ApJ, 704, 1120

White, B., \& Sofia, U. J. 2011, BAAS, 218, 12923

Wyse, A. B. 1942, ApJ, 95, 356

Zeippen, C. J. 1982, MNRAS, 198, 111 